Dedicated to the centenary of the publication of the Painlevé VI equation in the Comptes Rendus de l'Academie des Sciences de Paris

by Richard Fuchs in 1905.

\title{
RANDOM MATRIX THEORY AND THE SIXTH PAINLEVÉ EQUATION
}

\author{
P.J. FORRESTER AND N.S. WITTE
}

\begin{abstract}
A feature of certain ensembles of random matrices is that the corresponding measure is invariant under conjugation by unitary matrices. Study of such ensembles realised by matrices with Gaussian entries leads to statistical quantities related to the eigenspectrum, such as the distribution of the largest eigenvalue, which can be expressed as multidimensional integrals or equivalently as determinants. These distributions are well known to be $\tau$ functions for Painlevé systems, allowing for the former to be characterised as the solution of certain nonlinear equations. We consider the random matrix ensembles for which the nonlinear equation is the $\sigma$ form of $\mathrm{P}_{\mathrm{VI}}$. Known results are reviewed, as is their implication by way of series expansions for the distributions. New results are given for the boundary conditions in the neighbourhood of the fixed singularities at $t=0,1, \infty$ of $\sigma \mathrm{P}_{\mathrm{VI}}$ displayed by a generalisation of the generating function for the distributions. The structure of these expansions is related to Jimbo's general expansions for the $\tau$-function of $\sigma \mathrm{P}_{\mathrm{VI}}$ in the neighbourhood of its fixed singularities, and this theory is itself put in its context of the linear isomonodromy problem relating to $\mathrm{P}_{\mathrm{VI}}$.
\end{abstract}

\section{INTRODUCTION}

1.1. The $\sigma$-form of Painlevé VI. Given a large sequence of an eigenvalue spectrum, it is a simple matter to rescale so that the mean spacing between consecutive eigenvalues is unity, then to empirically determine the distribution function for the spacing. When these eigenspectra are the highly excited states of heavy nuclei, it is a celebrated result that the distribution function is well approximated by the functional form

$$
p_{1}^{(\mathrm{W})}(s):=\frac{\pi s}{2} e^{-\pi s^{2} / 4},
$$

known as the Wigner surmise. It is in the exact computation of eigenvalues distributions for certain classical random matrix ensembles that Painlevé transcendents make their appearance. These transcendents may relate to any of $\mathrm{P}_{\mathrm{II}}$ to $\mathrm{P}_{\mathrm{VI}}$, depending on the random matrix ensemble under consideration, or its scaled limits.

2000 Mathematics Subject Classification. 05E35, 39A05, 37F10, 33C45, 34M55. 
As part of the centenary of the discovery of Painlevé VI by Fuchs [11], in this paper we will restrict attention to the random matrix ensembles relating to $\mathrm{P}_{\mathrm{VI}}$.

Painlevé VI conventionally refers to the four parameter second order nonlinear differential equation

$$
\begin{aligned}
y^{\prime \prime}=\frac{1}{2}\left(\frac{1}{y}+\frac{1}{y-1}\right. & \left.+\frac{1}{y-t}\right)\left(y^{\prime}\right)^{2}-\left(\frac{1}{t}+\frac{1}{t-1}+\frac{1}{y-t}\right) y^{\prime} \\
& +\frac{y(y-1)(y-t)}{t^{2}(t-1)^{2}}\left(\alpha+\frac{\beta t}{y^{2}}+\frac{\gamma(t-1)}{(y-1)^{2}}+\frac{\delta t(t-1)}{(y-t)^{2}}\right),
\end{aligned}
$$

as obtained by Fuchs [11]. However in random matrix theory this equation is never encountered directly. Rather what is encountered is the so-called Jimbo-MiwaOkamoto $\sigma$-form of Painlevé VI,

$$
\sigma_{\mathrm{VI}}^{\prime}\left(t(t-1) \sigma_{\mathrm{VI}}^{\prime \prime}\right)^{2}+\left(\sigma_{\mathrm{VI}}^{\prime}\left[2 \sigma_{\mathrm{VI}}-(2 t-1) \sigma_{\mathrm{VI}}^{\prime}\right]+v_{1} v_{2} v_{3} v_{4}\right)^{2}=\prod_{k=1}^{4}\left(\sigma_{\mathrm{VI}}^{\prime}+v_{k}^{2}\right) .
$$

This is written in a form which displays a $D_{4}$ root system symmetry in the parameters $v_{1}, \ldots, v_{4}$. When expanded out there is a common factor of $\sigma_{\mathrm{VI}}^{\prime}$, which when cancelled out shows (1.3) to be a second order second degree nonlinear differential equation. The equations (1.2) and (1.3) are related by the Hamiltonian formulation of $\mathrm{P}_{\mathrm{VI}}$, due originally to Malmquist in 1922 [19].

In the Hamiltonian approach to the Painlevé equations in general, one presents a Hamiltonian $H\left(q, p, t ;\left\{v_{k}\right\}\right)$ where $\left\{v_{k}\right\}$ are parameters, such that after eliminating $p$ in the Hamilton equations

$$
q^{\prime}=\frac{\partial H}{\partial p}, \quad p^{\prime}=-\frac{\partial H}{\partial q},
$$

where the dash denotes derivatives with respect to $t$, the equation in $q$ is the appropriate Painlevé equation. The Hamiltonians can be systematically derived from the isomonodromy deformation theory associated with the Painlevé equations [16], [22] (aspects of the isomonodromy deformation theory associated with $\mathrm{P}_{\mathrm{VI}}$ is covered in Section 3 below). From such considerations, the Hamiltonian relating to $\mathrm{P}_{\mathrm{VI}}$ was given by Okamoto [23] as

$$
\begin{aligned}
& t(t-1) H_{\mathrm{VI}}=q(q-1)(q-t) p^{2} \\
& \begin{aligned}
-\left[\left(v_{3}+v_{4}\right)(q-1)(q-t)+\left(v_{3}-v_{4}\right) q(q-t)\right. & \left.-\left(v_{1}+v_{2}\right) q(q-1)\right] p \\
& +\left(v_{3}-v_{1}\right)\left(v_{3}-v_{2}\right)(q-t)
\end{aligned}
\end{aligned}
$$

where the the parameters $v_{1}, \ldots, v_{4}$ are related to $\alpha, \beta, \gamma, \delta$ in (1.2) according to

$\alpha=\frac{1}{2}\left(v_{1}-v_{2}\right)^{2}, \quad \beta=-\frac{1}{2}\left(v_{3}+v_{4}\right)^{2}, \quad \gamma=\frac{1}{2}\left(v_{3}-v_{4}\right)^{2}, \quad \delta=\frac{1}{2}\left(1-\left(1-v_{1}-v_{2}\right)^{2}\right)$,

and $q$ satisfies (1.2). Note that $H_{\mathrm{VI}}$ is quadratic in $p$ and thus according to the first of the Hamilton equations (1.4) $p$ can be written as a rational function of $q$ 
and $q^{\prime}$, and thus in fact $H_{\mathrm{VI}}$ is a rational function in $q$ and $q^{\prime}$. According to the following result, this particular rational function, augmented by the addition of a linear function in $t$, satisfies (1.3) [23].

Proposition 1.1. Define the auxiliary Hamiltonian

$$
h_{\mathrm{VI}}(t)=t(t-1) H_{\mathrm{VI}}+e_{2}\left[-v_{1},-v_{2}, v_{3}\right] t-\frac{1}{2} e_{2}\left[-v_{1},-v_{2}, v_{3}, v_{4}\right],
$$

where

$$
e_{p}\left[a_{1}, \ldots, a_{s}\right]:=\sum_{1 \leq j_{1}<\ldots<j_{p} \leq s} a_{j_{1}} a_{j_{2}} \cdots a_{j_{p}} .
$$

This auxiliary Hamiltonian satisfies the $\sigma$-form of Painlevé VI (1.3).

1.2. Historical Overview. There are certain ensembles of $N \times N$ random matrices with complex Gaussian entries and invariance under conjugation by unitary matrices, which have their joint eigenvalue probability distribution function (p.d.f.) of the form

$$
\frac{1}{C} \prod_{l=1}^{N} w_{2}\left(x_{l}\right) \prod_{1 \leq j<k \leq N}\left(x_{k}-x_{j}\right)^{2}
$$

where the weight function $w_{2}(x)$ is of one of the classical forms

$$
w_{2}(x)= \begin{cases}e^{-x^{2}}, & \text { Gaussian } \\ x^{a} e^{-x} \quad(x>0), & \text { Laguerre } \\ x^{a}(1-x)^{b} \quad(0<x<1), & \text { Jacobi }\end{cases}
$$

For example, let $X$ be a $n \times N(n \geq N)$ rectangular matrix of complex Gaussians $\mathrm{N}\left[0,1 / \sqrt{(2)]}+i \mathrm{~N}\left[0,1 / \sqrt{(2)]}\right.\right.$. Then the matrix $X^{\dagger} X$ has eigenvalue p.d.f. (1.9) with Laguerre weight $x^{n-N} e^{-x} \quad(x>0)$. The sixth Painlevé equation relates to (1.9) with Jacobi weight; in particular to the probability that there are exactly $n$ eigenvalues in the interval $(t, 1)$ of that ensemble. This probability is in turn equal to the coefficient of $(1-\xi)^{n}$ in the expansion of

$$
\begin{aligned}
E_{N}^{J}(t ; a, b ; \xi):=\frac{1}{C}\left(\int_{0}^{1}-\xi \int_{t}^{1}\right) d & x_{1} \cdots\left(\int_{0}^{1}-\xi \int_{t}^{1}\right) d x_{N} \\
& \times \prod_{l=1}^{N} x_{l}^{a}\left(1-x_{l}\right)^{b} \prod_{1 \leq j<k \leq N}\left(x_{k}-x_{j}\right)^{2},
\end{aligned}
$$

where $C$ denotes the normalisation. In the case $N=1$ (1.11) is an integral form of a particular ${ }_{2} F_{1}$ hypergeometric function, which was related to the Painlevé VI equation by Okamoto [23].

Let $\left\{p_{j}(x)\right\}_{j=0,1, \ldots}$ denote the set of monic polynomials of degree $j$ orthogonal with respect to the Jacobi weight $x^{a}(1-x)^{b}(0<x<1)$, which are given in terms 
of the Jacobi polynomials $P_{j}^{(a, b)}$ by

$$
p_{j}(x)=(-1)^{j} j ! \frac{\Gamma(a+b+j+1)}{\Gamma(a+b+2 j+1)} P_{j}^{(a, b)}(1-2 x) .
$$

Let $\left(p_{j}, p_{j}\right)_{2}:=\int_{0}^{1}\left(p_{j}(x)\right)^{2} x^{a}(1-x)^{b} d x$, and define

$$
\tilde{K}_{N}^{J}(x, y)=\frac{\left(w_{2}(x) w_{2}(y)\right)^{1 / 2}}{\left(p_{N-1}, p_{N-1}\right)_{2}} \frac{p_{N}(x) p_{N-1}(y)-p_{N-1}(x) p_{N}(y)}{x-y} .
$$

It is well known, and easy to derive (see e.g. [4]), that with $\tilde{K}_{N,(t, 1)}^{J}$ denoting the integral operator on $(t, 1)$ with kernel (1.13),

$$
E_{N}^{J}(t ; a, b ; \xi)=\operatorname{det}\left(1-\xi \tilde{K}_{N,(t, 1)}^{J}\right) .
$$

It was in this form that

$$
t(t-1) \frac{d}{d t} \log E_{N}^{J}
$$

was first related to the solution of a nonlinear equation by Tracy and Widom [24]. The equation found was of third order. Subsequently Haine and Semengue [13] studied (1.11) itself in the case $\xi=1$ and found a different third order equation for (1.15). Upon subtracting the two equations they obtained a second order second degree nonlinear equation which they identified as an example of the $\sigma$-form of Painlevé VI (1.3). The study of Tracy and Widom proceeded via functional properties of quantities associated with the Fredholm determinant (1.14), while Haine and Semengue used the theory of the KP hierarchy and Virasoro constraints satisfied by certain matrix integrals as introduced by Adler and van Moerbeke [1]. A third approach to the problem was initiated by Borodin and Deift [3]. They combined Riemann-Hilbert theory with the method of isomonodromic deformation of certain linear differential equations to obtain a characterisation of (1.15) which allows for immediate identification with the parameters in (1.3) (this is not the case with [13]). Explicitly it was shown that

$$
\sigma(t)=-t(t-1) \frac{d}{d t} \log E_{N}^{J}(1-t ; a, b ; \xi)+v_{1} v_{2} t+\frac{1}{2}\left(-v_{1} v_{2}+v_{3} v_{4}\right),
$$

satisfies (1.3) with

$$
v_{1}=v_{2}=N+\frac{a+b}{2}, \quad v_{3}=\frac{a+b}{2}, \quad v_{4}=\frac{a-b}{2} .
$$

Furthermore, it is required that as $t \rightarrow 0$

$$
\begin{aligned}
\frac{d}{d t} \log E_{N}^{J}(1-t ; a, b ; \xi) & \sim-\xi \tilde{K}_{N}^{J}(1-t, 1-t) \\
& =-\xi C_{N}(a, b)(1-t)^{b}
\end{aligned}
$$

where

$$
C_{N}(a, b)=\frac{\Gamma(a+b+N+1) \Gamma(b+N+1)}{\Gamma(N) \Gamma(a+N) \Gamma(b+1) \Gamma(b+2)},
$$

thus providing the boundary condition to be satisfied by (1.16). 
According to (1.16)

$$
E_{N}^{J}(1-t ; a, b ; \xi)=\exp \int_{1-t}^{1} \frac{d s}{s(1-s)}\left(\sigma(s)-v_{1} v_{2} s-\frac{1}{2}\left(-v_{1} v_{2}+v_{3} v_{4}\right)\right)
$$

From the characterisation of $\sigma(t)$ as a $\sigma \mathrm{P}_{\mathrm{VI}}$ transcendent with a specific boundary condition, the power series solution of (1.21) about $t=1$ can be readily computed [4],

$$
E_{N}^{J}(1-t ; a, b ; \xi)=1
$$

$$
\begin{aligned}
& -\xi \frac{C_{N}(a, b)}{b+1}(1-t)^{b+1}\left\{1-\frac{(b+1)\left(2 N^{2}+2(a+b) N-2-2 b+a b\right)}{(b+2)^{2}}(1-t)+\mathrm{O}\left((1-t)^{2}\right)\right\} \\
& +\xi^{2} \frac{C_{N}^{2}(a, b)(N-1)(N+b+1)(N+a-1)(N+a+b+1)}{(b+2)^{2}\left(b^{2}+4 b+3\right)^{2}}(1-t)^{2 b+4}\{1+\mathrm{O}(1-t)\} .
\end{aligned}
$$

Furthermore, one can anticipate from (1.14) that the leading term in $1-t$ accompanying the power $\xi^{k}$ will be proportional to $(1-t)^{k b+k^{2}}$, as is consistent with (1.22).

It is well known (see e.g. [4]) that after the change of variables $x_{j}=\cos ^{2} \theta_{j} / 2,0 \leq$ $\theta_{j}<\pi$ and with $a, b= \pm 1 / 2$ the eigenvalue p.d.f for the Jacobi ensemble as specified by (1.9) and (1.10) becomes identical to the eigenvalue p.d.f. for matrices from the classical groups $O^{ \pm}(N), S p(2 N)$ chosen with Haar (uniform) measure. As a consequence (in an obvious notation)

$$
\begin{aligned}
& E^{O^{-}(2 N+1)}((0, \phi) ; \xi)=\left.E_{N}^{J}\left(\cos ^{2} \phi / 2 ; \xi\right)\right|_{\substack{a=1 / 2 \\
b=-1 / 2}} \\
& E^{O^{+}(2 N+1)}((0, \phi) ; \xi)=\left.E_{N}^{J}\left(\cos ^{2} \phi / 2 ; \xi\right)\right|_{\substack{a=-1 / 2 \\
b=1 / 2}} .
\end{aligned}
$$

Analogous to the expansion (1.22), the $\sigma \mathrm{P}_{\mathrm{VI}}$ evaluation (1.21) can then be used to deduce the expansions [4]

$$
\begin{gathered}
\text { (1.24) } E^{O^{-}(2 N+1)}((0, x) ; \xi)=1-\tilde{c} x+\frac{4 N^{2}-1}{36} \tilde{c} x^{3} \\
-\frac{48 N^{4}-40 N^{2}+7}{3600} \tilde{c} x^{5}+\frac{4 N^{4}-5 N^{2}+1}{2025} \tilde{c}^{2} x^{6} \\
+\frac{192 N^{6}-336 N^{4}+196 N^{2}-31}{211680} \tilde{c} x^{7}-\frac{48 N^{6}-112 N^{4}+77 N^{2}-13}{198450} \tilde{c}^{2} x^{8}+\mathrm{O}\left(x^{9}\right),
\end{gathered}
$$

$$
\begin{aligned}
& E^{O^{+}(2 N+1)}((0, x) ; \xi)=1-\frac{4 N^{2}-1}{36} \tilde{c} x^{3} \\
+ & \frac{\left(4 N^{2}-1\right)\left(12 N^{2}-7\right)}{3600} \tilde{c} x^{5}-\frac{\left(4 N^{2}-1\right)\left(48 N^{4}-72 N^{2}+31\right)}{211680} \tilde{c} x^{7}+\mathrm{O}\left(x^{9}\right),
\end{aligned}
$$

where $\tilde{c}=2 N \xi / \pi$. 
The method of [24] was adopted in [25] to relate

$$
\begin{aligned}
E_{N}^{C y}(s ; \eta ; \xi):=\frac{1}{C}\left(\int_{-\infty}^{\infty}-\xi \int_{s}^{\infty}\right) d & x_{1} \cdots\left(\int_{-\infty}^{\infty}-\xi \int_{s}^{\infty}\right) d x_{N} \\
& \times \prod_{l=1}^{N} \frac{1}{\left(1+x_{l}^{2}\right)^{\eta}} \prod_{1 \leq j<k \leq N}\left(x_{k}-x_{j}\right)^{2},
\end{aligned}
$$

to $\sigma \mathrm{P}_{\mathrm{VI}}$. Explicitly it was shown that

$$
\sigma(s)=\left(1+s^{2}\right) \frac{d}{d s} \log E_{N}^{C y}(s ; a+N ; \xi),
$$

satisfies the equation

$$
\begin{aligned}
& \left(1+s^{2}\right)^{2}\left(\sigma^{\prime \prime}\right)^{2}+4\left(1+s^{2}\right)\left(\sigma^{\prime}\right)^{3}-8 s \sigma\left(\sigma^{\prime}\right)^{2}+4 \sigma^{2}\left(\sigma^{\prime}-a^{2}\right) \\
& +8 a^{2} s \sigma \sigma^{\prime}+4\left[N(N+2 a)-a^{2} s^{2}\right]\left(\sigma^{\prime}\right)^{2}=0 .
\end{aligned}
$$

As noted in [7], the relationship between (1.28) and (1.3) can be seen by changing variables

$$
t \mapsto \frac{i s+1}{2}, \quad \sigma_{\mathrm{VI}}(t) \mapsto \frac{i}{2} h(s),
$$

in the latter so that it reads

$$
h^{\prime}\left(\left(1+s^{2}\right) h^{\prime \prime}\right)^{2}+4\left(h^{\prime}\left(h-s h^{\prime}\right)-i v_{1} v_{2} v_{3} v_{4}\right)^{2}+4 \prod_{k=1}^{4}\left(h^{\prime}+v_{k}^{2}\right)=0 .
$$

With

$$
h=\sigma-a^{2} s, \quad v_{1}=-a, \quad v_{2}=0, \quad v_{3}=N+a, \quad v_{4}=a,
$$

(1.30) reduces to $(1.28)$.

The interest in (1.26) in random matrix theory comes about by making a stereographic projection from the real line to the unit circle by the change of variables $x=\tan \theta / 2$. With $z=e^{i \theta}$ this shows

$$
\begin{aligned}
\prod_{l=1}^{N} \frac{1}{\left(1+x_{l}^{2}\right)^{N+\eta}} & \prod_{1 \leq j<k \leq N}\left(x_{k}-x_{j}\right)^{2} d x_{1} \cdots d x_{N} \\
= & 2^{-N(N+2 \eta)} \prod_{l=1}^{N}\left|1+z_{l}\right|^{2 \eta} \prod_{1 \leq j<k \leq N}\left|z_{k}-z_{j}\right|^{2} d \theta_{1} \cdots d \theta_{N} .
\end{aligned}
$$

In the case $\eta=0$ the measure on the right hand side of (1.32) corresponds to the eigenvalue p.d.f. for $N \times N$ random unitary matrices chosen with Haar (uniform) measure. For general $\eta \in \mathbb{N}$ it corresponds to this same ensemble conditional so that there is an eigenvalue of degeneracy $\eta$ at $\theta=\pi$.

With $E_{N}^{U(N)}\left(\left(\phi_{1}, \phi_{2}\right) ; \xi\right)$ denoting the generating function for the probability that the interval $\left(\phi_{1}, \phi_{2}\right)$ contains exactly $n$ eigenvalues, it follows that

$$
E_{N}^{U(N)}((0,2 x) ; \xi)=\exp \left(-\int_{0}^{x} h(\cot \phi) d \phi\right)
$$


where $h(s)$ satisfies (1.30) with $v_{1}=v_{2}=v_{3}=0, v_{4}=N$. Since for $x \rightarrow 0$, $E_{N}^{U(N)}((0,2 x) ; \xi) \sim 1-\xi x N / \pi$, we seek the solution of (1.30) subject to the boundary condition $h(s) \sim c, c:=\xi N / \pi$. This allows the power series expansion

$$
\begin{aligned}
& E_{N}^{U(N)}((0,2 x) ; \xi)=1-c x+\frac{N^{2}-1}{36} c^{2} x^{4}-\frac{\left(N^{2}-1\right)\left(2 N^{2}-3\right)}{1350} c^{2} x^{6} \\
& +\frac{\left(N^{2}-1\right)\left(N^{2}-2\right)\left(3 N^{2}-5\right)}{52920} c^{2} x^{8}-\frac{\left(N^{2}-4\right)\left(N^{2}-1\right)^{2}}{291600} c^{3} x^{9}+\mathrm{O}\left(x^{10}\right),
\end{aligned}
$$

to be computed [4]. This expansion was first computed in [24] using the characterisation of $E_{N}^{U(N)}$ in terms of a third order nonlinear differential equation.

We have given reference to three distinct approaches which relate (1.11) to nonlinear differential equations with the Painlevé property. There is a fourth approach, which is due to the present authors [5], [6] [7], and involves applying Okamoto's theory of the Hamiltonian systems approach to $\mathrm{P}_{\mathrm{VI}}$ [23]. This approach has the advantage of allowing generalisations of the generating functions (1.11), (1.26) and $E_{N}^{U(N)}\left(\left(\phi_{1}, \phi_{2}\right) ; \xi\right)$ to be related to $\sigma \mathrm{P}_{\mathrm{VI}}$. Consider for definiteness the latter. In [7] the more general quantity

$$
\begin{aligned}
A_{N}\left(t ; \omega_{1}, \omega_{2}, \mu ; \xi^{*}\right):= & \frac{1}{N !}\left(\int_{-\pi}^{\pi}-\xi^{*} \int_{\pi-\phi}^{\pi}\right) \frac{d \theta_{1}}{2 \pi} \ldots\left(\int_{-\pi}^{\pi}-\xi^{*} \int_{\pi-\phi}^{\pi}\right) \frac{d \theta_{N}}{2 \pi} \\
& \times \prod_{l=1}^{N} z_{l}^{-i \omega_{2}}\left|1+z_{l}\right|^{2 \omega_{1}}\left|1+t z_{l}\right|^{2 \mu} \prod_{1 \leq j<k \leq N}\left|z_{j}-z_{k}\right|^{2}
\end{aligned}
$$

where $t=e^{i \phi}, \phi \in[0,2 \pi), \xi^{*} \in \mathbb{C}$ and the parameters $\omega_{1}, \omega_{2}, \mu \in \mathbb{C}, \omega=\omega_{1}+i \omega_{2}$, are restricted with $\Re\left(2 \omega_{1}\right), \Re(2 \mu)>-1, N \in \mathbb{Z}_{\geq 0}$. The independent variable $t$, whilst originally defined on the unit circle $|t|=1$ with a real angle $\phi$, can be considered as a complex variable which is analytically continued into the cut complex $t$-plane. The case $\omega_{2}=\mu=0, \omega_{1}=\eta$ of (1.35) gives the generating function for the probability of $k$ eigenvalues in $(0, \phi)$ for the ensemble specified by the right hand side of (1.32). Define

$$
\begin{aligned}
M_{N}(a, b) & :=\int_{-1 / 2}^{1 / 2} d x_{1} \ldots \int_{-1 / 2}^{1 / 2} d x_{N} \prod_{l=1}^{N} z_{l}^{(a-b) / 2}\left|1+z_{l}\right|^{a+b} \prod_{1 \leq j<k \leq N}\left|z_{j}-z_{k}\right|^{2} \\
& =\prod_{j=0}^{N-1} \frac{\Gamma(a+b+j+1) \Gamma(j+2)}{\Gamma(a+j+1) \Gamma(b+j+1)}, \quad z_{l}=e^{2 \pi i x_{l}}, l=1, \ldots, N,
\end{aligned}
$$

set $\xi^{*}=1-(1-\xi) e^{-\pi i \mu}$ and denote by $\tilde{h}(s)$ a solution of (1.30) with

$$
v_{1}=-\mu-\omega_{1}, \quad v_{2}=i \omega_{2}, \quad v_{3}=N+\mu+\omega_{1}, \quad v_{4}=-\mu+\omega_{1} .
$$


It was shown in [7] that

$$
\begin{aligned}
& A_{N}\left(t ; \omega_{1}, \omega_{2}, \mu ; \xi^{*}\right)=\frac{M_{N}(\mu+\bar{\omega}, \mu+\omega)}{M_{N}(0,0)} \\
& \quad \times \exp \left(-\frac{1}{2} \int_{0}^{\phi}\left[\tilde{h}\left(\cot \frac{\theta}{2}\right)+\omega_{2}\left(N+\omega_{1}-\mu\right)+\left(\mu+\omega_{1}\right)^{2} \cot \frac{\theta}{2}\right] d \theta\right) .
\end{aligned}
$$

We remark that according to the theory in the sentence including (1.30)

$$
\tilde{h}\left(\cot \frac{\theta}{2}\right)=-2 i \sigma_{\mathrm{VI}}\left(\frac{1}{1+e^{i \theta}}\right) .
$$

A generalisation of the Jacobi ensemble (1.11) may also be made in a similar fashion, by the introduction of the additional factor $\prod_{l=1}^{N}\left|s-x_{l}\right|^{\mu}$ in the integrand. In the case $\xi=0$ it is shown in [7] that this generalised ensemble can be related to (1.35), and consequently that

$$
\begin{aligned}
\sigma_{\mathrm{VI}}(t)=\left(e_{2}\left[-v_{1},-v_{2}, v_{3}\right]+N \mu\right) & t-\frac{1}{2} e_{2}\left[-v_{1},-v_{2}, v_{3}, v_{4}\right]-N \mu \\
& +t(t-1) \frac{d}{d t} \log A_{N}\left(t ; \omega_{1}, \omega_{2}, \mu ; \xi^{*}=0\right),
\end{aligned}
$$

is a solution of (1.3) with the parameters

$v_{1}=\frac{N+\omega-\mu}{2}, \quad v_{2}=\bar{\omega}+\frac{N+\omega+\mu}{2}, \quad v_{3}=\frac{N-\omega+\mu}{2}, \quad v_{4}=-\mu-\frac{N+\omega+\mu}{2}$.

Moreover a derivation of the transformation implied by (1.40) and (1.41) was given which tells us that the condition $\xi^{*}=0$ in (1.41) can be relaxed.

The above mentioned generalisation of the Jacobi ensemble (1.11) has also revealed different Painlevé connections to those found in [7]. In the mid 1990's, with $\xi=0$, this was studied from the viewpoint of orthogonal polynomial theory by Magnus [18]. In that study an auxiliary quantity occurring in the theory was shown to satisfy the $\mathrm{P}_{\text {VI }}$ equation (1.2) for appropriate parameters. We remark that further development of orthogonal polynomial theory in relation to $(1.35)[9,8]$ has been shown to relate to Bäcklund transformations in the Hamiltonian theory of $\mathrm{P}_{\mathrm{VI}}$, and to the so called discrete $\mathrm{dP}_{\mathrm{V}}$ equations.

In the case $\omega_{2}=\mu=0, \omega_{1}=\eta(1.35)$ is the generating function for the probability that there are exactly $n$ eigenvalues in $(\pi-\phi, \pi)$ for the eigenvalue p.d.f. on the right hand side of (1.35). In this case expanding (1.14) allow us to specify the $s \rightarrow \infty$ boundary condition which must be satisfied by $\tilde{h}(s)$. However, for generic parameters the boundary conditions were not given in [7]. Consideration of this latter problem is our concern for the remainder of the paper. A similar situation was recently rectified in [10] in relation to a class of multidimensional integral solutions of $\sigma \mathrm{P}_{\mathrm{V}}$ [6]. The approach taken was to write the multidimensional integral as a determinant (involving the confluent hypergeometric function), and to expand the entries of the determinant. An analogous strategy suffices in relation to (1.35), 
now with the entries of the determinant given in terms of the Gauss hypergeometric function. This is done in Section 2.

The form of the general expansion of a solution of the $\sigma \mathrm{P}_{\mathrm{VI}}$ equation (1.3), or equivalently its $\tau$ function about its fixed singularities $t=0,1, \infty$ has been given by Jimbo [15]. The exponents occurring therein are given in terms of the monodromy data associated with the isomonodromy deformation formulation of $\mathrm{P}_{\mathrm{VI}}$. Aspects of this theory and the results of Jimbo are revised in Section 3.

One of the features of the general expansions is that they consist of two branches, whereas the expansion of (1.35) only exhibits a single branch. We show in Section 4 that this is entirely consistent with the monodromy data associated with $\sigma_{\mathrm{VI}}$; it is such that the coefficient in front of one of the branches vanishes identically.

\section{Boundary Conditions}

Consider the multidimensional integral (1.35). According to (1.40) (with the condition $\xi^{*}=0$ relaxed), we know how to relate its logarithmic derivative to a solution of the $\sigma \mathrm{P}_{\mathrm{VI}}$ equation (1.3). However this property cannot be used to characterise the multidimensional integral unless an appropriate boundary condition is specified. As remarked in the second paragraph below (1.42) above, in [7] the interpretation of (1.35) for some special parameters as the generating function of a gap

probability provided the boundary condition in those cases. However for general parameters no boundary condition was presented.

Taking a different approach, namely the expansion of the elements in the determinant form of (1.35), the sought boundary conditions can be deduced as presented in the following result.

Proposition 2.1. For generic values of $\mu, \omega, \bar{\omega}$ the spectral average $A_{N}$ has the following expansions. About $t=0$ subject to $\mu-\bar{\omega} \notin \mathbb{Z}$ we have

$$
\begin{aligned}
t^{N \mu} A_{N} \underset{t \rightarrow 0}{\sim}\left\{1+\xi^{*} \frac{e^{-\pi i(\mu-\bar{\omega})}}{2 i \sin \pi(\mu-\bar{\omega})}\right\}^{N} \prod_{k=0}^{N-1} \frac{k ! \Gamma\left(2 \omega_{1}+k+1\right)}{\Gamma(1+k+\mu+\omega) \Gamma(1+k-\mu+\bar{\omega})} \\
\times\left\{1+\frac{2 N \mu(\mu+\omega)}{N-\mu+\bar{\omega}} t\right. \\
\quad-\frac{\xi^{*} e^{-\pi i(\mu-\bar{\omega})}}{2 i \sin \pi(\mu-\bar{\omega})+\xi^{*} e^{-\pi i(\mu-\bar{\omega})}} \\
\left.\times \frac{\Gamma(1+\mu+\omega) \Gamma(1+\mu-\bar{\omega}) \Gamma(1+2 \mu) \Gamma(N-\mu+\bar{\omega})}{\Gamma(N) \Gamma(N+\mu+\bar{\omega}) \Gamma\left(N+2 \omega_{1}\right) \Gamma^{2}(2-N+\mu-\bar{\omega})} t^{1-N+\mu-\bar{\omega}}\right\} .
\end{aligned}
$$


About $t=1$ subject to $2 \mu+2 \omega_{1} \notin \mathbb{Z}$ we have

$$
\begin{gathered}
A_{N} \underset{t \rightarrow 1}{\sim} \prod_{k=0}^{N-1} \frac{k ! \Gamma\left(2 \mu+2 \omega_{1}+k+1\right)}{\Gamma(1+k+\mu+\omega) \Gamma(1+k+\mu+\bar{\omega})}\left\{1+\frac{N \mu(\bar{\omega}-\omega)}{2 \mu+2 \omega_{1}}(1-t)\right. \\
+\frac{(-1)^{N+1}}{\sin \pi\left(2 \mu+2 \omega_{1}\right)}\left(\xi^{*} \frac{e^{-\pi i(\mu-\bar{\omega})}}{2 i}+\frac{\sin \pi 2 \mu \sin \pi(\mu+\omega)}{\sin \pi\left(2 \mu+2 \omega_{1}\right)}\right) \\
\left.\times \frac{\Gamma(1+2 \mu) \Gamma\left(1+2 \omega_{1}\right) \Gamma(1+\mu+\omega) \Gamma(1+\mu+\bar{\omega})}{\Gamma^{2}\left(2 \mu+2 \omega_{1}+2\right) \Gamma\left(2 \mu+2 \omega_{1}+1\right) \Gamma(N) \Gamma\left(-N-2 \mu-2 \omega_{1}\right)}(1-t)^{1+2 \mu+2 \omega_{1}}\right\} .
\end{gathered}
$$

And about $t=\infty$ subject to $\mu-\omega \notin \mathbb{Z}$ we have

$$
\begin{aligned}
& t^{-\mu N} A_{N} \underset{t \rightarrow \infty}{\sim}\left\{-\frac{e^{-\pi i 2 \mu}}{\sin \pi(\mu-\omega)}\left(\sin \pi(\mu+\omega)+\xi^{*} \frac{e^{-\pi i(\mu+\omega)}}{2 i}\right)\right\}^{N} \\
& \times \prod_{k=0}^{N-1} \frac{k ! \Gamma\left(2 \omega_{1}+k+1\right)}{\Gamma(1+k+\mu+\bar{\omega}) \Gamma(1+k-\mu+\omega)} \\
& \times\left\{1+\frac{2 \mu N(\mu+\bar{\omega})}{N-\mu+\omega} \frac{1}{t}\right. \\
&+ e^{\pi i(\mu-\omega)}\left(\frac{2 i \sin \pi 2 \mu+\xi^{*} e^{-\pi i 2 \mu}}{2 i \sin \pi(\mu+\omega)+\xi^{*} e^{-\pi i(\mu+\omega)}}\right) \\
&\left.\times \frac{\Gamma(1+2 \mu) \Gamma(1+\mu+\bar{\omega}) \Gamma(1+\mu-\omega) \Gamma(N-\mu+\omega)}{\Gamma(N) \Gamma(N+\mu+\omega) \Gamma\left(N+2 \omega_{1}\right) \Gamma^{2}(2-N+\mu-\omega)} t^{-1+N-\mu+\omega}\right\}
\end{aligned}
$$

Any one of these boundary conditions suffices to uniquely define a solution to the ordinary differential equation (1.3) under the above generic restrictions on the parameters.

Proof. Using Heine's identity we can deduce from (1.35) that

$$
\begin{aligned}
& A_{N}\left(t ; \omega_{1}, \omega_{2}, \mu ; \xi^{*}\right) \\
= & t^{-N \mu} \operatorname{det}\left[\left(\int_{-\pi}^{\pi}-\xi^{*} \int_{\pi-\phi}^{\pi}\right) \frac{d \theta}{2 \pi} z^{-\mu-\omega+j-k}(1+z)^{2 \omega_{1}}(1+t z)^{2 \mu}\right]_{0 \leq j, k \leq N-1} .
\end{aligned}
$$

This is a determinant of a Toeplitz matrix whose symbol, or more specifically weight $w(z)$, is one defined on the unit circle $|z|=1$ by

$$
w(z)=t^{-\mu} z^{-\mu-\omega}(1+z)^{2 \omega_{1}}(1+t z)^{2 \mu}\left\{\begin{array}{l}
1, \quad \theta \in(-\pi, \pi-\phi) \\
1-\xi^{*}, \quad \theta \in(\pi-\phi, \pi)
\end{array},\right.
$$

with Fourier components $w_{k}$ such that $w(z)=\sum_{k=-\infty}^{\infty} w_{k} z^{k}$. Considering the expansion about $t=0$ first we note that the Toeplitz matrix element can be evaluated 
as

$$
\begin{aligned}
& t^{\mu} w_{n}=\{1+\left.\xi^{*} \frac{e^{-\pi i(n+\mu-\bar{\omega})}}{2 i \sin \pi(n+\mu-\bar{\omega})}\right\} \frac{\Gamma\left(2 \omega_{1}+1\right)}{\Gamma(1+n+\mu+\omega) \Gamma(1-n-\mu+\bar{\omega})} \\
& \times{ }_{2} F_{1}(-2 \mu,-n-\mu-\omega ; 1-n-\mu+\bar{\omega} ; t) \\
&-\xi^{*} \frac{e^{-\pi i(n+\mu-\bar{\omega})}}{2 i \sin \pi(n+\mu-\bar{\omega})} \frac{\Gamma(2 \mu+1)}{\Gamma(1+n+\mu-\bar{\omega}) \Gamma(1-n+\mu+\bar{\omega})} \\
& \quad \times t^{n+\mu-\bar{\omega}}{ }_{2} F_{1}\left(-2 \omega_{1}, n-\mu-\bar{\omega} ; 1+n+\mu-\bar{\omega} ; t\right),
\end{aligned}
$$

under the condition that $n+\mu-\bar{\omega} \notin \mathbb{Z}$, which is a form suitable for the development of an expansion about $t=0$. In the course of the derivation we had to invoke tighter constraints on the parameters, namely $|t|=1, t \neq 1$ and $\Re(2 \mu) \geq 0, \Re\left(2 \omega_{1}\right) \geq 0$, but these can be relaxed by analytic continuation arguments. The structure is $t^{\mu} w_{n}=a_{n}(t)+t^{n+\mu-\bar{\omega}} b_{n}(t)$ where $a_{n}(t), b_{n}(t)$ are analytic about $t=0$. One can expand the Toeplitz determinant about $t=0$ retaining only the leading order terms from both the analytic and non-analytic contributions and the resulting formula is

$$
\begin{aligned}
t^{\mu N} A_{N}(t) \underset{t \rightarrow 0}{\sim} \operatorname{det}\left(a_{j-k}(0)+t a_{j-k}^{\prime}(0)\right)_{j, k=0, \ldots, N-1} \\
\quad+(-1)^{N-1} b_{-(N-1)} \operatorname{det}\left(a_{j-k+1}(0)\right)_{j, k=0, \ldots, N-2} t^{\mu-\bar{\omega}-(N-1)}
\end{aligned}
$$

Using the determinant identity [21]

$$
\operatorname{det}\left(\frac{\Gamma(d+k-j)}{\Gamma(c+k-j)}\right)_{0 \leq j, k \leq n-1}=\prod_{j=0}^{n-1} j ! \frac{\Gamma(1+d-c)}{\Gamma(1+d-c-j)} \frac{\Gamma(d-n+1+j)}{\Gamma(c+j)}
$$

or its slightly more general form

$$
\begin{aligned}
\operatorname{det}\left(\frac{\Gamma\left(z_{k}+b-j\right)}{\Gamma\left(z_{k}-j\right)}\right)_{0 \leq j, k \leq n-1} & \\
= & \prod_{0 \leq j<k \leq n-1}\left(z_{k}-z_{j}\right) \prod_{j=0}^{n-1}(-1)^{j}(-b)_{j} \frac{\Gamma\left(z_{j}+b-n+1\right)}{\Gamma\left(z_{j}\right)},
\end{aligned}
$$

for an arbitrary sequence $\left\{z_{j}\right\}_{j=0}^{n-1}$ one can evaluate the determinants appearing in (2.7). The result is $(2.1)$.

To develop the expansion about $t=1$ the appropriate expression for the Toeplitz matrix element is

$$
\begin{aligned}
& t^{\bar{\omega}-n} w_{n}=\frac{\Gamma\left(2 \mu+2 \omega_{1}+1\right)}{\Gamma(1+n+\mu+\omega) \Gamma(1-n+\mu+\bar{\omega})} \\
& \quad \times{ }_{2} F_{1}\left(-2 \omega_{1}, n-\mu-\bar{\omega} ;-2 \mu-2 \omega_{1} ; 1-t\right) \\
& +\frac{1}{\pi}\left(\xi^{*} \frac{e^{-\pi i(n+\mu-\bar{\omega})}}{2 i}+\frac{\sin \pi 2 \mu \sin \pi(n+\mu+\omega)}{\sin \pi\left(2 \mu+2 \omega_{1}\right)}\right) \frac{\Gamma(1+2 \mu) \Gamma\left(1+2 \omega_{1}\right)}{\Gamma\left(2+2 \mu+2 \omega_{1}\right)} \\
& \quad \times(1-t)^{1+2 \mu+2 \omega_{1}}{ }_{2} F_{1}\left(1+2 \mu, n+1+\mu+\omega ; 2+2 \mu+2 \omega_{1} ; 1-t\right),
\end{aligned}
$$


subject to $1+2 \mu+2 \omega_{1} \notin \mathbb{Z}$. The structure is now $t^{\bar{\omega}-n} w_{n}=a_{n}(1-t)+(1-$ $t)^{1+2 \mu+2 \omega_{1}} b_{n}(1-t)$ where $a_{n}(1-t), b_{n}(1-t)$ are analytic about $t=1$. Again one can expand the Toeplitz determinant about $t=1$ retaining only the leading order terms from both the analytic and non-analytic contributions and use the above determinant identities to arrive at (2.2).

Lastly the expansion about $t=\infty$ is computed using the Toeplitz matrix element in the form

$$
\begin{aligned}
& t^{\mu} w_{n}=\frac{e^{-\pi i 2 \mu}}{\sin \pi(n-\mu+\omega)}\left(\sin \pi(n+\mu+\omega)+\xi^{*} \frac{e^{-\pi i(n+\mu+\omega)}}{2 i}\right) \\
& \quad \times \frac{\Gamma\left(2 \omega_{1}+1\right)}{\Gamma(1+n-\mu+\omega) \Gamma(1-n+\mu+\bar{\omega})} \\
& \times t^{2 \mu}{ }_{2} F_{1}(-2 \mu, n-\mu-\bar{\omega} ; 1+n-\mu+\omega ; 1 / t) \\
& -\frac{e^{-\pi i(n+\mu+\omega)}}{\sin \pi(n-\mu+\omega)}\left(\begin{array}{c}
\left.\sin \pi 2 \mu+\xi^{*} \frac{e^{-\pi i 2 \mu}}{2 i}\right) \frac{\Gamma(2 \mu+1)}{\Gamma(1+n+\mu+\omega) \Gamma(1-n+\mu-\omega)} \\
\quad \times t^{n+\mu+\omega}{ }_{2} F_{1}\left(-2 \omega_{1},-n-\mu-\omega ; 1-n+\mu-\omega ; 1 / t\right),
\end{array}\right.
\end{aligned}
$$

valid for $n-\mu+\omega \notin \mathbb{Z}$. The structure is $t^{-\mu} w_{n}=a_{n}(1 / t)+t^{n-\mu+\omega} b_{n}(1 / t)$ where $a_{n}(1 / t), b_{n}(1 / t)$ are analytic about $t=\infty$. Repeating the procedure adopted about the other singularities one arrives at (2.3).

Remark 2.1. We do not attempt to treat the degenerate cases where either $\mu-\bar{\omega} \in$ $\mathbb{Z}, 2 \mu+2 \omega_{1} \in \mathbb{Z}$ or $\mu-\omega \in \mathbb{Z}$ here as this results in confluent logarithms and other technical difficulties.

\section{Isomonodromy Deformation Formulation for P Pi}

In this section we describe the isomonodromic deformation system which characterises the general solution to the sixth Painlevé equation and outline a solution to the direct monodromy problem, that is explicit formulae for the monodromy data associated with a particular solution of the $\sigma$-form for the sixth Painlevé equation. The isomonodromic deformation system associated with the sixth Painlevé equation is not uniquely determined and we shall see this arbitrariness arise in Section 4 when applying the general theory to our random matrix theory.

Following the conventions and notations of [15], [17] we consider the $2 \times 2$ linear matrix ODE for $\Psi(\lambda ; t)$ with four regular singularities in the $\lambda$-plane chosen to be $\nu=0, t, 1, \infty$

$$
\begin{aligned}
\frac{\partial}{\partial \lambda} \Psi & =\left(\frac{A_{0}}{\lambda}+\frac{A_{1}}{\lambda-1}+\frac{A_{t}}{\lambda-t}\right) \Psi, \\
\frac{\partial}{\partial t} \Psi & =-\frac{A_{t}}{\lambda-t} \Psi .
\end{aligned}
$$


It is taken that the residue matrices $A_{\nu}(t)$ satisfy

$$
A_{0}+A_{t}+A_{1}=-A_{\infty}=-\frac{\theta_{\infty}}{2} \sigma_{3}, \quad \sigma_{3}:=\left(\begin{array}{cc}
1 & 0 \\
0 & -1
\end{array}\right), \quad \theta_{\infty} \in \mathbb{C} \backslash \mathbb{Z}
$$

and given the freedom to choose either $\operatorname{tr} A_{\nu}$ or one of the eigenvalues we follow the convention of Jimbo [15]

$$
\operatorname{tr} A_{\nu}=0, \quad \operatorname{det} A_{\nu}=-\frac{1}{4} \theta_{\nu}^{2}, \nu=0, t, 1, \infty,
$$

defining the formal exponents of monodromy $\theta_{\nu}$. The residue matrices satisfy the Schlesinger system of equations

$$
\begin{aligned}
\frac{d}{d t} A_{0} & =-\frac{\left[A_{0}, A_{t}\right]}{t}, \\
\frac{d}{d t} A_{t} & =\frac{\left[A_{0}, A_{t}\right]}{t}+\frac{\left[A_{t}, A_{1}\right]}{1-t}, \\
\frac{d}{d t} A_{1} & =-\frac{\left[A_{t}, A_{1}\right]}{1-t}, \\
\frac{d}{d t} A_{\infty} & =0
\end{aligned}
$$

as a consequence of the compatibility of (3.1) and (3.2).

The $\tau$-function for $\mathrm{P}_{\mathrm{VI}}$ is defined by

$$
\frac{d}{d t} \log \tau=\operatorname{Tr}\left(\frac{A_{0}}{t}+\frac{A_{1}}{t-1}\right) A_{t}
$$

and the $\sigma$-function

$$
\zeta(t)=t(t-1) \frac{d}{d t} \log \tau+\frac{1}{4}\left(\theta_{t}^{2}-\theta_{\infty}^{2}\right) t-\frac{1}{8}\left(\theta_{t}^{2}+\theta_{0}^{2}-\theta_{\infty}^{2}-\theta_{1}^{2}\right)
$$

which satisfies the second-order second degree differential equation

$$
\begin{aligned}
\frac{d}{d t} \zeta\left(t(t-1) \frac{d^{2}}{d t^{2}} \zeta\right)^{2} & \\
+\left[2 \frac{d}{d t} \zeta\left(t \frac{d}{d t} \zeta-\zeta\right)\right. & \left.-\left(\frac{d}{d t} \zeta\right)^{2}-\frac{1}{16}\left(\theta_{t}^{2}-\theta_{\infty}^{2}\right)\left(\theta_{0}^{2}-\theta_{1}^{2}\right)\right]^{2} \\
= & \left(\frac{d}{d t} \zeta+\frac{1}{4}\left(\theta_{t}+\theta_{\infty}\right)^{2}\right)\left(\frac{d}{d t} \zeta+\frac{1}{4}\left(\theta_{t}-\theta_{\infty}\right)^{2}\right) \\
& \times\left(\frac{d}{d t} \zeta+\frac{1}{4}\left(\theta_{0}+\theta_{1}\right)^{2}\right)\left(\frac{d}{d t} \zeta+\frac{1}{4}\left(\theta_{0}-\theta_{1}\right)^{2}\right)
\end{aligned}
$$

(cf. $(1.3))$.

Furthermore we suppose (Assumption 1) that the matrices $A_{\nu}$ are diagonalisable, i.e. that there exists nonsingular $R_{\nu} \in S L(2, \mathbb{C})$ such that

$$
R_{\nu}^{-1} A_{\nu} R_{\nu}=\frac{1}{2} \theta_{\nu} \sigma_{3}, \theta_{\nu} \in \mathbb{C} \backslash \mathbb{Z}
$$


In the neighbourhood of a regular singularity $\Psi(\lambda)$ can be expanded locally as

$$
\Psi=\sum_{m=0}^{\infty} \Psi_{m \nu}(\lambda-\nu)^{m+\frac{\theta_{\nu}}{2} \sigma_{3}} C_{\nu},
$$

for $\nu=0, t, 1$ and for $\lambda=\infty$ in the form

$$
\Psi=\left(I+\sum_{m=1}^{\infty} \Psi_{m \infty} \lambda^{-m}\right) \lambda^{-\frac{\theta_{\infty}}{2} \sigma_{3}} .
$$

For such local series to exist we have to assume (Assumption 2) that the eigenvalues of $A_{\nu}$ are distinct modulo the non-zero integers, i.e. that $\pm \theta_{\nu} \notin \mathbb{N}$.

The matrices $C_{\nu}, \nu=0, t, 1$ are the connection matrices and we are taking the local solutions (3.14) as our fundamental system of solutions, i.e. $C_{\infty}=I$. The monodromy matrices $M_{\nu}(\nu=0, t, 1, \infty)$ are defined as

$$
\left.\Psi\right|_{\lambda=\nu+\delta e^{2 \pi i}}=\left.\Psi\right|_{\lambda=\nu+\delta} M_{\nu}, \quad \nu=0, t, 1, \infty
$$

and are given in terms of the monodromy exponents and connection matrices by

$$
M_{\nu}=C_{\nu}^{-1} e^{\pi i \theta_{\nu} \sigma_{3}} C_{\nu} .
$$

The monodromy matrices together satisfy the cyclic relation

$$
M_{\infty} M_{1} M_{t} M_{0}=I
$$

according to the convention taken for the basis of loops displayed in Figure 1, which generate the fundamental group $\pi\left(\mathbb{C P} \backslash\{0, t, 1, \infty\}, \lambda_{0}\right)$. There is arbitrariness in the monodromy data in the sense that the replacement $C_{\nu} \mapsto D_{\nu}^{-1} C_{\nu}$ doesn't change the monodromy matrices provided that $D_{\nu}$ commutes with the right-hand side of (3.12). This implies that $D_{\nu}$ is diagonal if $\theta_{\nu} \neq 0$. This arbitrariness will manifest itself in the appearance of an arbitrary complex number in the explicit parameterisation discussed latter. 


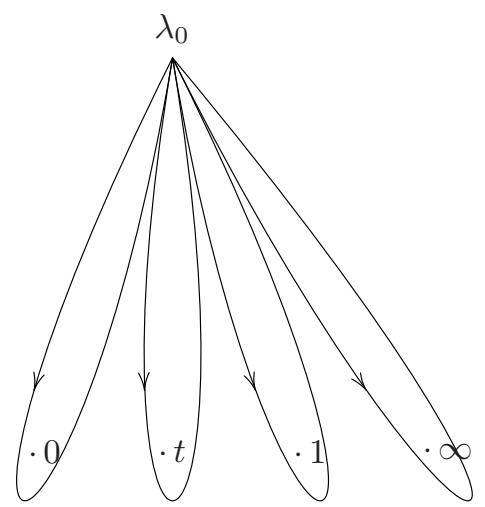

FiguRE 1. Monodromy representation of the fundamental group for $\mathbb{C P} \backslash\{0, t, 1, \infty\}$

The isomonodromic principle states that the monodromy data $M D:=\left\{\theta_{\nu}, C_{\nu}, M_{\nu} \mid \nu=\right.$ $0, t, 1, \infty\}$ are preserved under the deformations of $t$. The invariants of the monodromy data are defined

$$
\begin{aligned}
p_{\mu} & =2 \cos \pi \theta_{\mu}:=\operatorname{Tr} M_{\mu} \quad \mu \in\{0, t, 1, \infty\} \\
p_{\mu \nu} & =2 \cos \pi \sigma_{\mu \nu}:=\operatorname{Tr} M_{\mu} M_{\nu} \quad \mu, \nu \in\{0, t, 1\},
\end{aligned}
$$

in the sense that these do not contain any arbitrary constants.

In [15] Jimbo states the following conditions under which his results apply

$$
\begin{aligned}
& \theta_{0}, \theta_{t}, \theta_{1}, \theta_{\infty} \notin \mathbb{Z}, \\
& 0<\Re\left(\sigma_{0 t}\right)<1, \\
& \theta_{0} \pm \theta_{t} \pm \sigma_{0 t}, \quad \theta_{\infty} \pm \theta_{1} \pm \sigma_{0 t} \notin 2 \mathbb{Z} .
\end{aligned}
$$

When $\sigma_{0 t} \neq 0$ a parameterisation of the monodromy matrices was deduced by Jimbo and is given in Lemma 3.1. 
Lemma 3.1 (Jimbo[15]). Subject to the conditions (3.20), (3.21) and (3.22) the monodromy matrices can be parameterised in the following way

$$
M_{\infty}=\left(\begin{array}{cc}
e^{\pi i \theta_{\infty}} & 0 \\
0 & e^{-\pi i \theta_{\infty}}
\end{array}\right)
$$

$$
M_{1}=\frac{1}{i \sin \pi \theta_{\infty}}\left(\begin{array}{c}
\cos \pi \sigma-e^{-\pi i \theta_{\infty}} \cos \pi \theta_{1} \\
2 r^{-1} e^{\pi i \theta_{\infty}} \sin \frac{\pi}{2}\left(\theta_{\infty}-\theta_{1}+\sigma\right) \sin \frac{\pi}{2}\left(\theta_{\infty}-\theta_{1}-\sigma\right)
\end{array}\right.
$$$$
\begin{gathered}
-2 r e^{-\pi i \theta_{\infty}} \sin \frac{\pi}{2}\left(\theta_{\infty}+\theta_{1}+\sigma\right) \sin \frac{\pi}{2}\left(\theta_{\infty}+\theta_{1}-\sigma\right) \\
-\cos \pi \sigma+e^{\pi i \theta_{\infty}} \cos \pi \theta_{1}
\end{gathered}
$$

$$
C M_{t} C^{-1}=\frac{1}{i \sin \pi \sigma}\left(\begin{array}{c}
e^{\pi i \sigma} \cos \pi \theta_{t}-\cos \pi \theta_{0} \\
2 s^{-1} e^{-\pi i \sigma} \sin \frac{\pi}{2}\left(\theta_{0}+\theta_{t}+\sigma\right) \sin \frac{\pi}{2}\left(\theta_{0}-\theta_{t}-\sigma\right)
\end{array}\right.
$$

$$
\left.\begin{array}{c}
-2 s e^{\pi i \sigma} \sin \frac{\pi}{2}\left(\theta_{0}+\theta_{t}-\sigma\right) \sin \frac{\pi}{2}\left(\theta_{0}-\theta_{t}+\sigma\right) \\
-e^{-\pi i \sigma} \cos \pi \theta_{t}+\cos \pi \theta_{0}
\end{array}\right),
$$

$$
C M_{0} C^{-1}=\frac{1}{i \sin \pi \sigma}\left(\begin{array}{c}
e^{\pi i \sigma} \cos \pi \theta_{0}-\cos \pi \theta_{t} \\
-2 s^{-1} \sin \frac{\pi}{2}\left(\theta_{0}-\theta_{t}-\sigma\right) \sin \frac{\pi}{2}\left(\theta_{0}+\theta_{t}+\sigma\right)
\end{array}\right.
$$$$
\left.\begin{array}{c}
2 s \sin \frac{\pi}{2}\left(\theta_{0}+\theta_{t}-\sigma\right) \sin \frac{\pi}{2}\left(\theta_{0}-\theta_{t}+\sigma\right) \\
-e^{-\pi i \sigma} \cos \pi \theta_{0}+\cos \pi \theta_{t}
\end{array}\right),
$$

where

$$
C=\left(\begin{array}{cc}
\sin \frac{\pi}{2}\left(\theta_{\infty}-\theta_{1}-\sigma\right) & r \sin \frac{\pi}{2}\left(\theta_{\infty}+\theta_{1}+\sigma\right) \\
r^{-1} \sin \frac{\pi}{2}\left(\theta_{\infty}-\theta_{1}+\sigma\right) & \sin \frac{\pi}{2}\left(\theta_{\infty}+\theta_{1}-\sigma\right)
\end{array}\right)
$$

Here $r$ is an arbitrary non-zero complex number, and the short hand notation $s:=s_{0 t}, \sigma=\sigma_{0 t}$ is used. 
Proof. We begin by noting that (3.23) follows from our choice for the fundamental system of solutions. To establish the other formula we require two preliminary results. Let us make the abbreviations $c_{\nu}:=\cos \pi \theta_{\nu}, s_{\nu}:=\sin \pi \theta_{\nu}, c_{\sigma}:=$ $\cos \pi \sigma_{0 t}, s_{\sigma}:=\sin \pi \sigma_{0 t}, \epsilon_{\nu}:=c_{\nu}+i s_{\nu}$ and

$$
\mathfrak{S}(\vartheta):=\sin \frac{\pi}{2} \vartheta
$$

and note some trigonometric identities which will be useful in this and the ensuing proofs

$$
\begin{aligned}
2 \mathfrak{S}\left(\theta_{\infty}-\theta_{1}+\sigma\right) \mathfrak{S}\left(\theta_{\infty}+\theta_{1}+\sigma\right) & =c_{1}-c_{\infty} c_{\sigma}+s_{\infty} s_{\sigma}, \\
2 \mathfrak{S}\left(\theta_{\infty}+\theta_{1}-\sigma\right) \mathfrak{S}\left(\theta_{\infty}-\theta_{1}-\sigma\right) & =c_{1}-c_{\infty} c_{\sigma}-s_{\infty} s_{\sigma}, \\
2 \mathfrak{S}\left(\theta_{\infty}-\theta_{1}+\sigma\right) \mathfrak{S}\left(\theta_{\infty}-\theta_{1}-\sigma\right) & =c_{\sigma}-c_{1} c_{\infty}-s_{1} s_{\infty}, \\
2 \mathfrak{S}\left(\theta_{\infty}+\theta_{1}+\sigma\right) \mathfrak{S}\left(\theta_{\infty}+\theta_{1}-\sigma\right) & =c_{\sigma}-c_{1} c_{\infty}+s_{1} s_{\infty}, \\
2 \mathfrak{S}\left(\theta_{\infty}-\theta_{1}+\sigma\right) \mathfrak{S}\left(\theta_{\infty}+\theta_{1}-\sigma\right) & =-c_{\infty}+c_{1} c_{\sigma}+s_{1} s_{\sigma}, \\
2 \mathfrak{S}\left(\theta_{\infty}+\theta_{1}+\sigma\right) \mathfrak{S}\left(\theta_{\infty}-\theta_{1}-\sigma\right) & =-c_{\infty}+c_{1} c_{\sigma}-s_{1} s_{\sigma}, \\
2 \mathfrak{S}\left(\theta_{0}-\theta_{t}+\sigma\right) \mathfrak{S}\left(\theta_{0}+\theta_{t}+\sigma\right) & =c_{t}-c_{0} c_{\sigma}+s_{0} s_{\sigma}, \\
2 \mathfrak{S}\left(\theta_{0}+\theta_{t}-\sigma\right) \mathfrak{S}\left(\theta_{0}-\theta_{t}-\sigma\right) & =c_{t}-c_{0} c_{\sigma}-s_{0} s_{\sigma}, \\
2 \mathfrak{S}\left(\theta_{0}-\theta_{t}+\sigma\right) \mathfrak{S}\left(\theta_{0}+\theta_{t}-\sigma\right) & =-c_{0}+c_{t} c_{\sigma}+s_{t} s_{\sigma}, \\
2 \mathfrak{S}\left(\theta_{0}+\theta_{t}+\sigma\right) \mathfrak{S}\left(\theta_{0}-\theta_{t}-\sigma\right) & =-c_{0}+c_{t} c_{\sigma}-s_{t} s_{\sigma} .
\end{aligned}
$$

We also note that a general member $M$ of $\mathrm{SL}(2, \mathbb{C})$, characterised by $\operatorname{det} M=1$ and $\operatorname{tr} M=2 c$, can be written as

$$
M=\left(\begin{array}{cc}
c+x & -(s+i x) b \\
\frac{s-i x}{b} & c-x
\end{array}\right)
$$

where $c^{2}+s^{2}=1$, and with arbitrary complex numbers $b, b^{-1} \neq 0, x^{-1} \neq 0$. One can parameterise $c=\cos \pi \theta, s=\sin \pi \theta$. The observation made in [2] that simplifies the derivation is that the Jimbo parameterisation is constructed so that

$$
C M_{t} M_{0} C^{-1}=\Delta:=\left(\begin{array}{cc}
\epsilon_{\sigma} & 0 \\
0 & \epsilon_{\sigma}^{-1}
\end{array}\right)
$$

for a non-singular matrix $C \in \mathrm{GL}(2, \mathbb{C})$. This implies

$$
C M_{1}^{-1} M_{\infty}^{-1} C^{-1}=\left(\begin{array}{cc}
\epsilon_{\sigma} & 0 \\
0 & \epsilon_{\sigma}^{-1}
\end{array}\right) \text {. }
$$

Using (3.23) and the parameterisation (3.39) for $M_{1}$ we can recast the above relation as a homogeneous matrix equation for $C$, that is

$$
\left(\begin{array}{cc}
\epsilon_{\sigma} & 0 \\
0 & \epsilon_{\sigma}^{-1}
\end{array}\right) C=C\left(\begin{array}{cc}
\epsilon_{\infty}\left(c_{1}+x\right) & -\epsilon_{\infty}\left(s_{1}+i x\right) b \\
\frac{s_{1}-i x}{b \epsilon_{\infty}} & \frac{c_{1}-x}{\epsilon_{\infty}}
\end{array}\right) .
$$


This has a solution if and only if $i x s_{\infty}=c_{\sigma}-c_{1} c_{\infty}$, which fixes $x$. Using the identities $(3.29,3.30,3.31,3.32)$ the equation for the components of $C$ can be written

$$
\begin{array}{r}
-i \epsilon_{\infty} b \mathfrak{S}\left(\theta_{\infty}+\theta_{1}+\sigma\right) C_{11}+\mathfrak{S}\left(\theta_{\infty}-\theta_{1}-\sigma\right) C_{12}=0 \\
\epsilon_{\infty} b \mathfrak{S}\left(\theta_{\infty}+\theta_{1}-\sigma\right) C_{21}+i \mathfrak{S}\left(\theta_{\infty}-\theta_{1}+\sigma\right) C_{22}=0
\end{array}
$$

assuming $\theta_{\infty} \pm \theta_{1} \pm \sigma \neq \mathbb{Z}$. If one defines an arbitrary complex constant $r:=i b \epsilon_{\infty}$ then this yields the solution (3.27) for $C$. We note that $\operatorname{det} C=-s_{\infty} s_{\sigma}$. Employing the solution for $x$ and the identities $(3.31,3.32)$ we arrive at the formula $(3.24)$ for $M_{1}$. For the remaining monodromy matrices we utilise the representation (3.39) for $C M_{0} C^{-1}$ and $C M_{t} C^{-1}$ in the relation (3.40). From the $(1,1)$ and $(2,2)$ components of the resulting matrix equation we find

$$
\begin{aligned}
\epsilon_{\infty} c_{0}-c_{t} & =\epsilon_{\infty} x_{0}+x_{t}, \\
-\epsilon_{\infty}^{-1} c_{0}+c_{t} & =\epsilon_{\infty}^{-1} x_{0}+x_{t},
\end{aligned}
$$

and one deduces that $i s_{\sigma} x_{0}=c_{0} c_{\sigma}-c_{t}$ and $i s_{\sigma} x_{t}=c_{t} c_{\sigma}-c_{0}$. From the other components we find that $x_{t}^{2}-x_{0}^{2}=c_{t}^{2}-c_{0}^{2}$ and the formula for the ratio of the other undetermined constants is given by

$$
\frac{b_{t}}{b_{0}}=\epsilon_{\sigma} \frac{\mathfrak{S}\left(\theta_{0}-\theta_{t}-\sigma\right)}{\mathfrak{S}\left(\theta_{0}-\theta_{t}+\sigma\right)}
$$

where we have utilised $(3.35,3.36,3.37,3.38)$. If we define the remaining undetermined constant $b_{t}:=-i \epsilon_{\sigma} s_{0 t}$ then we recover the parameterisations (3.26), (3.25) for $M_{0}, M_{t}$.

A key identity is the following connection relation which relates $s_{0 t}, \sigma_{0 t}$ to $\sigma_{t 1}$ and $\sigma_{01}$.

Lemma 3.2 (Jimbo[15]). One of the connection relations is

$$
\begin{aligned}
& 4 s_{0 t}^{ \pm 1} \sin \frac{\pi}{2}\left(\theta_{0}+\theta_{t} \mp \sigma_{0 t}\right) \sin \frac{\pi}{2}\left(\theta_{0}-\theta_{t} \pm \sigma_{0 t}\right) \\
& \quad \times \sin \frac{\pi}{2}\left(\theta_{\infty}+\theta_{1} \mp \sigma_{0 t}\right) \sin \frac{\pi}{2}\left(\theta_{\infty}-\theta_{1} \pm \sigma_{0 t}\right) \\
& =e^{ \pm \pi i \sigma_{0 t}}\left( \pm i \sin \pi \sigma_{0 t} \cos \pi \sigma_{t 1}-\cos \pi \theta_{t} \cos \pi \theta_{\infty}-\cos \pi \theta_{0} \cos \pi \theta_{1}\right) \\
& \quad \pm i \sin \pi \sigma_{0 t} \cos \pi \sigma_{01}+\cos \pi \theta_{t} \cos \pi \theta_{1}+\cos \pi \theta_{\infty} \cos \pi \theta_{0}
\end{aligned}
$$

Proof. The proof of this has been detailed in Boalch [2] with a typographical correction to the original formula in [15]. So we content ourselves with a brief summary of the steps involved. After noting (3.40) it was found that the monodromy invariants are more manageable in the forms

$$
\begin{aligned}
& p_{t 1}=\operatorname{tr}\left(C M_{\infty}^{-1} C^{-1} \Delta^{-1}\left(C M_{t} C^{-1}\right)\right), \\
& p_{01}=\operatorname{tr}\left(C M_{\infty}^{-1} C^{-1} \Delta^{-1}\left(C M_{0} C^{-1}\right)\right),
\end{aligned}
$$


because they were linear in $1, s_{0 t}, s_{0 t}^{-1}$. Upon taking the combination $(3.49)+\epsilon_{\sigma}(3.50)$ in order to eliminate the $s_{0 t}^{-1}$ terms it was found that the resulting expression could be factorised through the use of the identities

$$
\begin{aligned}
& \mathfrak{S}\left(\theta_{\infty}+\theta_{1}-\sigma\right) \mathfrak{S}\left(\theta_{\infty}-\theta_{1}-\sigma\right) \\
& -\mathfrak{S}\left(\theta_{\infty}-\theta_{1}+\sigma\right) \mathfrak{S}\left(\theta_{\infty}+\theta_{1}+\sigma\right)=-s_{\infty} s_{\sigma}, \\
& \epsilon_{\infty}^{-1} \mathfrak{S}\left(\theta_{\infty}+\theta_{1}-\sigma\right) \mathfrak{S}\left(\theta_{\infty}-\theta_{1}-\sigma\right) \\
& -\epsilon_{\infty} \mathfrak{S}\left(\theta_{\infty}-\theta_{1}+\sigma\right) \mathfrak{S}\left(\theta_{\infty}+\theta_{1}+\sigma\right)=i s_{\infty}\left(\epsilon_{\sigma} c_{\infty}-c_{1}\right), \\
& \epsilon_{\infty} \mathfrak{S}\left(\theta_{\infty}+\theta_{1}-\sigma\right) \mathfrak{S}\left(\theta_{\infty}-\theta_{1}-\sigma\right) \\
& -\epsilon_{\infty}^{-1} \mathfrak{S}\left(\theta_{\infty}-\theta_{1}+\sigma\right) \mathfrak{S}\left(\theta_{\infty}+\theta_{1}+\sigma\right)=i s_{\infty}\left(c_{1}-\epsilon_{\sigma}^{-1} c_{\infty}\right),
\end{aligned}
$$

enabling a factor of $2 s_{\infty} s_{\sigma}$ to be cancelled out. This then yielded one of the desired formulae (3.48), whilst the other could be found from the other combination of $(3.49,3.50)$.

A consequence of this is a constraint on the monodromy invariants $\left\{p_{0 t}, p_{t 1}, p_{01}\right\}$ which is an algebraic variety defining a sub-manifold, the monodromy manifold, of $\mathbb{C}^{3}$.

Lemma 3.3 (Jimbo[15]). The monodromy manifold is given by

$$
\begin{array}{r}
p_{0 t} p_{t 1} p_{01}+p_{0 t}^{2}+p_{t 1}^{2}+p_{01}^{2}-\left(p_{0} p_{t}+p_{1} p_{\infty}\right) p_{0 t}-\left(p_{t} p_{1}+p_{0} p_{\infty}\right) p_{t 1}-\left(p_{0} p_{1}+p_{t} p_{\infty}\right) p_{01} \\
+p_{0}^{2}+p_{t}^{2}+p_{1}^{2}+p_{\infty}^{2}+p_{0} p_{t} p_{1} p_{\infty}-4=0 .
\end{array}
$$

Proof. We multiply the upper and lower sign forms of the left-hand side of (3.48) to eliminate $s_{0 t}$ and then employ the identities $(3.29,3.30,3.35,3.36)$ to replace the product of sines. Equating this to the corresponding product of the right-hand sides yields (3.54) as the only nontrivial factor.

Remark 3.1. The above connection relation involves only the free parameters $s_{0 t}$ and the monodromy invariants $\sigma_{0 t}, \sigma_{t 1}, \sigma_{01}$. As we shall see immediately below the arbitrary parameters $s_{0 t}$ and $\sigma_{0 t}$ appear in the expansion for the $\tau$-function about $t=0$, and there exist analogous pairs about $t=1, \infty$. Correspondingly there exist two other forms of the connection relation (3.48) involving either the parameters $s_{t 1}, s_{01}$ and can be deduced directly from (3.48) by a simple substitution rule given at the end of Theorem 3.2. However both connection relations yield the same formula (3.54) for the variety defining the monodromy manifold.

Now we come to the fundamental result for the expansion of the $\tau$-function in the neighbourhood of the fixed singularities of the sixth Painlevé system at $t=0,1, \infty$. 
Theorem 3.1 (Jimbo[15]). Under the conditions (3.20,3.21,3.22) we have the expansion of the $\tau$-function as $t \rightarrow 0$ in the domain $\{t \in \mathbb{C}|0<| t|<\varepsilon,| \arg (t) \mid<\phi\}$ for all $\varepsilon>0$ and any $\phi>0$

$$
\begin{aligned}
\tau(t) \sim C t^{\left(\sigma^{2}-\theta_{0}^{2}-\theta_{t}^{2}\right) / 4} & \\
& \times\left\{1+\frac{\left(\theta_{0}^{2}-\theta_{t}^{2}-\sigma^{2}\right)\left(\theta_{\infty}^{2}-\theta_{1}^{2}-\sigma^{2}\right)}{8 \sigma^{2}} t\right. \\
& -\hat{s} \frac{\left[\theta_{0}^{2}-\left(\theta_{t}-\sigma\right)^{2}\right]\left[\theta_{\infty}^{2}-\left(\theta_{1}-\sigma\right)^{2}\right]}{16 \sigma^{2}(1+\sigma)^{2}} t^{1+\sigma} \\
- & \left.\hat{s}^{-1} \frac{\left[\theta_{0}^{2}-\left(\theta_{t}+\sigma\right)^{2}\right]\left[\theta_{\infty}^{2}-\left(\theta_{1}+\sigma\right)^{2}\right]}{16 \sigma^{2}(1-\sigma)^{2}} t^{1-\sigma}+\mathrm{O}\left(|t|^{2(1-\Re(\sigma))}\right)\right\}
\end{aligned}
$$

where $\sigma \neq 0$ and $\hat{s}$ are related to $s$ through

$$
\begin{aligned}
\left.\hat{s}=s \frac{\Gamma^{2}(1-\sigma) \Gamma(1+}{\Gamma^{2}(1+\sigma) \Gamma(1+} \frac{1}{2}\left(\theta_{0}+\theta_{t}+\sigma\right)\right) \Gamma\left(1+\frac{1}{2}\left(-\theta_{0}+\theta_{t}+\sigma\right)\right) \\
\\
\times \frac{\Gamma\left(1+\frac{1}{2}\left(\theta_{\infty}+\theta_{1}+\sigma\right)\right) \Gamma\left(1+\frac{1}{2}\left(-\theta_{\infty}+\theta_{1}+\sigma\right)\right)}{\Gamma\left(1+\frac{1}{2}\left(\theta_{\infty}+\theta_{1}-\sigma\right)\right) \Gamma\left(1+\frac{1}{2}\left(-\theta_{\infty}+\theta_{1}-\sigma\right)\right)},
\end{aligned}
$$

and we employ the short-hand notation $s=s_{0 t}, \hat{s}=\hat{s}_{0 t}$ and $\sigma=\sigma_{0 t}$. The monodromy data defining the unique solution to the sixth Painlevé system is $\left\{\sigma_{0 t}, s_{0 t}\right\}$. Here $C$ is an arbitrary constant.

Proof. The details of this proof of this are given in Jimbo [15] and so we do not repeat them here. Also Guzzetti has laid out some of the intermediate steps in the appendix of his work on the elliptic representations of the general Painlevé six equation [12].

The regular singularities $x=0, t, 1, \infty$ play equivalent roles and can be exchanged under linear fractional or Möbius transformations. Consequently one can solve the connection problem very neatly and under the additional conditions

$$
\begin{aligned}
& 0<\Re\left(\sigma_{t 1}\right), \Re\left(\sigma_{01}\right)<1, \\
& \theta_{1} \pm \theta_{t} \pm \sigma_{t 1}, \quad \theta_{\infty} \pm \theta_{0} \pm \sigma_{t 1} \notin 2 \mathbb{Z}, \\
& \theta_{0} \pm \theta_{1} \pm \sigma_{01}, \quad \theta_{\infty} \pm \theta_{t} \pm \sigma_{01} \notin 2 \mathbb{Z},
\end{aligned}
$$

derive expansions about $t=1, \infty$. 
Theorem 3.2 (Jimbo[15]). Under the conditions (3.20,3.57,3.58) we have the expansion of the $\tau$-function as $t \rightarrow 1$

$$
\begin{array}{rl}
\tau(t) \sim C & C(1-t)^{\left(\sigma_{t 1}^{2}-\theta_{1}^{2}-\theta_{t}^{2}\right) / 4} \\
& \times\left\{1+\frac{\left(\theta_{1}^{2}-\theta_{t}^{2}-\sigma_{t 1}^{2}\right)\left(\theta_{\infty}^{2}-\theta_{0}^{2}-\sigma_{t 1}^{2}\right)}{8 \sigma_{t 1}^{2}}(1-t)\right. \\
- & \hat{s}_{t 1} \frac{\left[\theta_{1}^{2}-\left(\theta_{t}-\sigma_{t 1}\right)^{2}\right]\left[\theta_{\infty}^{2}-\left(\theta_{0}-\sigma_{t 1}\right)^{2}\right]}{16 \sigma_{t 1}^{2}\left(1+\sigma_{t 1}\right)^{2}}(1-t)^{1+\sigma_{t 1}} \\
\left.-\hat{s}_{t 1}^{-1} \frac{\left[\theta_{1}^{2}-\left(\theta_{t}+\sigma_{t 1}\right)^{2}\right]\left[\theta_{\infty}^{2}-\left(\theta_{0}+\sigma_{t 1}\right)^{2}\right]}{16 \sigma_{t 1}^{2}\left(1-\sigma_{t 1}\right)^{2}}(1-t)^{1-\sigma_{t 1}}+\mathrm{O}\left(|1-t|^{2\left(1-\Re\left(\sigma_{t 1}\right)\right)}\right)\right\},
\end{array}
$$

and as $t \rightarrow \infty$

$$
\begin{aligned}
& \tau(t) \sim C t^{-\left(\sigma_{01}^{2}-\theta_{\infty}^{2}+\theta_{t}^{2}\right) / 4} \\
& \times\left\{1+\frac{\left(\theta_{\infty}^{2}-\theta_{t}^{2}-\sigma_{01}^{2}\right)\left(\theta_{0}^{2}-\theta_{1}^{2}-\sigma_{01}^{2}\right)}{8 \sigma_{01}^{2}} t^{-1}\right. \\
&-\hat{s}_{01} \frac{\left[\theta_{\infty}^{2}-\left(\theta_{t}-\sigma_{01}\right)^{2}\right]\left[\theta_{0}^{2}-\left(\theta_{1}-\sigma_{01}\right)^{2}\right]}{16 \sigma_{01}^{2}\left(1+\sigma_{01}\right)^{2}} t^{-1-\sigma_{01}} \\
&\left.-\hat{s}_{01}^{-1} \frac{\left[\theta_{\infty}^{2}-\left(\theta_{t}+\sigma_{01}\right)^{2}\right]\left[\theta_{0}^{2}-\left(\theta_{1}+\sigma_{01}\right)^{2}\right]}{16 \sigma_{01}^{2}\left(1-\sigma_{01}\right)^{2}} t^{-1+\sigma_{01}}+\mathrm{O}\left(|t|^{-2\left(1-\Re\left(\sigma_{01}\right)\right)}\right)\right\} .
\end{aligned}
$$

Here $\hat{s}_{t 1}, \hat{s}_{01}$ are found by making the following substitutions in (3.56), (3.48) respectively

$$
\begin{aligned}
& \hat{s} \rightarrow \hat{s}_{t 1}, \quad s \rightarrow s_{t 1}, \quad \theta_{0} \leftrightarrow \theta_{1}, \quad \sigma \rightarrow \sigma_{t 1}, \quad \sigma_{t 1} \rightarrow \sigma_{0 t}, \\
& \hat{s} \rightarrow \hat{s}_{01}, \quad s \rightarrow s_{01}, \quad \theta_{0} \leftrightarrow \theta_{\infty}, \quad \sigma \rightarrow \sigma_{01}, \quad \sigma_{01} \rightarrow \tilde{\sigma}_{01},
\end{aligned}
$$

with

$\cos \pi \tilde{\sigma}_{01}=-\cos \pi \sigma_{0 t}-2 \cos \pi \sigma_{01} \cos \pi \sigma_{t 1}+2\left(\cos \pi \theta_{0} \cos \pi \theta_{t}+\cos \pi \theta_{\infty} \cos \pi \theta_{1}\right)$.

The monodromy data defining the unique solution to the sixth Painlevé system is either $\left\{\sigma_{t 1}, s_{t 1}\right\}$ or $\left\{\sigma_{01}, s_{01}\right\}$.

\section{Monodromy Data for the Spectrum Singularity Ensemble}

The precise relationship between the spectrum singularity average $A_{N}(t ;)$ and the isomonodromy theory of the sixth Painlevé system is given by the following result. Its validity relies on the conjecture that the expansions of Jimbo given in Theorems 3.1 and 3.2 remain valid upon relaxation of the constraints $(3.20,3.21,3.22)$ and $(3.57,3.58,3.59)$, provided the former are well defined (i.e. do not then diverge).

Proposition 4.1. For the spectrum singularity ensemble the associated isomonodromic system is not unique but the monodromy data for any of these systems 
falls into three generic cases. An example of each case is given below in cases (A), (B) and $(C)$. The formal monodromy exponents can be taken to belong to either of three sets

(4.1) $\operatorname{Case}(A): \quad \theta_{0}=-\mu-\omega, \quad \theta_{t}=N+2 \omega_{1}, \quad \theta_{1}=N+2 \mu, \quad \theta_{\infty}=-\mu-\bar{\omega}$,

(4.2) $\operatorname{Case}(B): \quad \theta_{0}=\mu-\bar{\omega}, \quad \theta_{t}=N, \quad \theta_{1}=N+2 \mu+2 \omega_{1}, \quad \theta_{\infty}=\mu-\omega$,

(4.3) $\operatorname{Case}(C): \quad \theta_{0}=-2 \omega_{1}, \quad \theta_{t}=N+\mu+\omega, \quad \theta_{1}=N+\mu+\bar{\omega}, \quad \theta_{\infty}=2 \mu$.

The monodromy invariants for either case are

$$
\sigma_{0 t}=N-\mu+\bar{\omega}, \quad \sigma_{t 1}=2 \mu+2 \omega_{1}, \quad \sigma_{01}=N-\mu+\omega .
$$

In the case (A) the monodromy coefficients are

$$
\begin{aligned}
& s_{0 t}=1+\frac{2 i \sin \pi(\mu-\bar{\omega})}{\xi^{*} e^{-\pi i(\mu-\bar{\omega})}}, \\
& s_{t 1}=1+\xi^{*} \frac{e^{-\pi i(\mu-\bar{\omega})}}{2 i} \frac{\sin \pi\left(2 \mu+2 \omega_{1}\right)}{\sin \pi 2 \mu \sin \pi(\mu+\omega)}, \\
& s_{01}=-\frac{\xi^{*}-1+e^{2 \pi i(\mu+\omega)}}{\xi^{*}-1+e^{4 \pi i \mu}} .
\end{aligned}
$$

All monodromy matrices are lower triangular

$$
\begin{gathered}
M_{0}=\left(\begin{array}{cc}
e^{-\pi i(\mu+\omega)} & 0 \\
m_{0} & e^{\pi i(\mu+\omega)}
\end{array}\right), \\
M_{t}=\left(\begin{array}{cc}
e^{\pi i\left(N+2 \omega_{1}\right)} & 0 \\
m_{t} & e^{-\pi i\left(N+2 \omega_{1}\right)}
\end{array}\right), \\
M_{1}=\left(\begin{array}{cc}
e^{\pi i(N+2 \mu)} & 0 \\
m_{1} & e^{-\pi i(N+2 \mu)}
\end{array}\right),
\end{gathered}
$$

where

$$
\begin{aligned}
& m_{0}=\frac{2 i}{\sin \pi(\mu-\bar{\omega})}\left\{\frac{\sin \pi 2 \omega_{1} \sin \pi(\mu+\bar{\omega})}{s_{0 t}}-\frac{\sin \pi 2 \mu \sin \pi(\mu+\omega)}{r}\right\} \\
& m_{t}=\frac{2 i(-1)^{N} \sin \pi 2 \omega_{1}}{\sin \pi(\mu-\bar{\omega})}\left\{-\frac{\sin \pi(\mu+\bar{\omega})}{s_{0 t}} e^{\pi i(\mu-\bar{\omega})}+\frac{\sin \pi 2 \mu}{r}\right\} \\
& m_{1}=-\frac{2 i(-1)^{N} \sin \pi 2 \mu}{r} e^{-\pi i(\mu+\bar{\omega})}
\end{aligned}
$$


For Case (B) the monodromy coefficients are

$s_{0 t}=1+\frac{2 i \sin \pi(\mu-\bar{\omega})}{\xi^{*} e^{-\pi i(\mu-\bar{\omega})}}$,

$$
s_{t 1} \frac{\sin \pi 2 \omega_{1} \sin \pi(\mu+\bar{\omega})}{\sin \pi\left(2 \mu+2 \omega_{1}\right)}=\frac{\sin \pi 2 \mu \sin \pi(\mu+\omega)}{\sin \pi\left(2 \mu+2 \omega_{1}\right)}+\xi^{*} \frac{e^{-\pi i(\mu-\bar{\omega})}}{2 i},
$$

$$
s_{01}=-\frac{\xi^{*}-1+e^{2 \pi i(\mu+\omega)}}{\xi^{*}-1+e^{4 \pi i \mu}} .
$$

One of monodromy matrices is proportional to the identity, the others are full

$$
M_{0}=\frac{i}{\sin \pi(\mu-\omega)}\left(\begin{array}{cc}
e^{-\pi i(\mu-\omega)} \cos \pi(\mu-\bar{\omega})-\cos \pi\left(2 \mu+2 \omega_{1}\right) & 2 r \sin \pi(\mu+\bar{\omega}) \sin \pi 2 \mu \\
-\frac{2}{r} \sin \pi(\mu+\omega) \sin \pi 2 \omega_{1} & -e^{\pi i(\mu-\omega)} \cos \pi(\mu-\bar{\omega})+\cos \pi\left(2 \mu+2 \omega_{1}\right)
\end{array}\right)
$$

$$
M_{t}=(-1)^{N} I \text {, }
$$

$$
M_{1}=\frac{i(-1)^{N}}{\sin \pi(\mu-\omega)}\left(\begin{array}{cc}
e^{-\pi i(\mu-\omega)} \cos \pi\left(2 \mu+2 \omega_{1}\right)-\cos \pi(\mu-\bar{\omega}) & 2 r e^{-\pi i(\mu-\omega)} \sin \pi(\mu+\bar{\omega}) \sin \pi 2 \mu \\
\frac{2}{r} e^{\pi i(\mu-\omega)} \sin \pi(\mu+\omega) \sin \pi 2 \omega_{1} & \cos \pi(\mu-\bar{\omega})-e^{\pi i(\mu-\omega)} \cos \pi\left(2 \mu+2 \omega_{1}\right)
\end{array}\right) .
$$


For Case (C) the monodromy coefficients are

$$
\begin{aligned}
s_{0 t} & =1+\frac{2 i \sin \pi(\mu-\bar{\omega})}{\xi^{*} e^{-\pi i(\mu-\bar{\omega})}} \\
s_{t 1} \frac{\sin \pi 2 \omega_{1} \sin \pi(\mu+\bar{\omega})}{\sin \pi\left(2 \mu+2 \omega_{1}\right)} & =\frac{\sin \pi 2 \mu \sin \pi(\mu+\omega)}{\sin \pi\left(2 \mu+2 \omega_{1}\right)}+\xi^{*} \frac{e^{-\pi i(\mu-\bar{\omega})}}{2 i}, \\
s_{01} & =-\frac{\xi^{*}-1+e^{2 \pi i(\mu+\omega)}}{\xi^{*}-1+e^{4 \pi i \mu}} .
\end{aligned}
$$

All monodromy matrices are upper triangular

$$
\begin{gathered}
M_{0}=\left(\begin{array}{cc}
e^{\pi i 2 \omega_{1}} & m_{0} \\
0 & e^{-\pi i 2 \omega_{1}}
\end{array}\right), \\
M_{t}=\left(\begin{array}{cc}
e^{-\pi i(N+\mu+\omega)} & m_{t} \\
0 & e^{\pi i(N+\mu+\omega)}
\end{array}\right), \\
M_{1}=\left(\begin{array}{cc}
e^{-\pi i(N+\mu+\bar{\omega})} & m_{1} \\
0 & e^{\pi i(N+\mu+\bar{\omega})}
\end{array}\right),
\end{gathered}
$$

where

$$
\begin{aligned}
& m_{0}=\frac{2 i}{\sin \pi(\mu-\bar{\omega})}\left\{-\sin \pi 2 \mu \sin \pi(\mu+\omega) s_{0 t}+\sin \pi 2 \omega_{1} \sin \pi(\mu+\bar{\omega}) r\right\} \\
& m_{t}=\frac{2 i(-1)^{N} \sin \pi(\mu+\omega)}{\sin \pi(\mu-\bar{\omega})}\left\{\sin \pi 2 \mu e^{-\pi i(\mu-\bar{\omega})} s_{0 t}-\sin \pi(\mu+\bar{\omega}) r\right\} \\
& m_{1}=2 i \sin \pi(\mu+\bar{\omega}) e^{-\pi i(N+2 \mu)} r .
\end{aligned}
$$

Proof. Comparison of the two differential equations for the $\sigma$-function, (1.3) and (3.11), imply that in general

$$
\left\{v_{1}, v_{2}, v_{3}, v_{4}\right\}=\frac{1}{2}\left\{\begin{array}{l}
\epsilon_{1}\left(\theta_{t}+\theta_{\infty}\right) \\
\epsilon_{2}\left(\theta_{t}-\theta_{\infty}\right) \\
\epsilon_{3}\left(\theta_{0}+\theta_{1}\right) \\
\epsilon_{4}\left(\theta_{0}-\theta_{1}\right)
\end{array},\right.
$$

with $\epsilon_{j}= \pm 1, j=1,2,3,4$ and $\epsilon_{1} \epsilon_{2} \epsilon_{3} \epsilon_{4}=1$. Using either set of parameters, (1.42) from the JUE correspondence or (1.38) from the CyUE correspondence, we find that the monodromy exponents can be given by one of three sets

$$
\left\{\theta_{0}, \theta_{t}, \theta_{1}, \theta_{\infty}\right\}=\left\{\begin{array}{l}
N+2 \mu, N+2 \omega_{1}, \mu+\omega, \mu+\bar{\omega} \\
N, N+2 \mu+2 \omega_{1}, \mu-\omega, \mu-\bar{\omega} \\
N+\mu+\omega, N+\mu+\bar{\omega},-2 \mu, 2 \omega_{1}
\end{array},\right.
$$

modulo permutations of the monodromy exponents and an even number of sign reversals. This is a manifestation of the non-uniqueness of the isomonodromic system for our problem. For definiteness we choose one example of the three cases, 
namely the cases (A), (B) and (C) given in $(4.1,4.2,4.3)$. We note some simple identities which do not depend on the choice of the permutation or the sign

$$
\begin{gathered}
v_{1}^{2}+v_{2}^{2}+v_{3}^{2}+v_{4}^{2}=\frac{1}{2}\left(\theta_{0}^{2}+\theta_{t}^{2}+\theta_{1}^{2}+\theta_{\infty}^{2}\right), \\
v_{1} v_{2} v_{3} v_{4}=\frac{1}{16}\left(\theta_{0}^{2}-\theta_{1}^{2}\right)\left(\theta_{t}^{2}-\theta_{\infty}^{2}\right) .
\end{gathered}
$$

and in particular the following products which apply equally to cases (A), (B) and

$$
\left(\theta_{0}^{2}-\theta_{1}^{2}\right)\left(\theta_{\infty}^{2}-\theta_{t}^{2}\right)=(N-\mu+\omega)(N+\mu-\omega)(N+3 \mu+\omega)(N+\mu+2 \bar{\omega}+\omega),
$$

$$
\begin{gathered}
\left(\theta_{0}^{2}-\theta_{t}^{2}\right)\left(\theta_{\infty}^{2}-\theta_{1}^{2}\right)=(N-\mu+\bar{\omega})(N+\mu-\bar{\omega})(N+3 \mu+\bar{\omega})(N+\mu+2 \omega+\bar{\omega}), \\
\left(\theta_{1}^{2}-\theta_{t}^{2}\right)\left(\theta_{\infty}^{2}-\theta_{0}^{2}\right)=\left(2 N+2 \mu+2 \omega_{1}\right)\left(2 \mu-2 \omega_{1}\right)\left(2 \mu+2 \omega_{1}\right)(\bar{\omega}-\omega) .
\end{gathered}
$$

If we make a comparison of the $\tau$-functions themselves for the JUE correspondence, (3.10) and (1.41) we find, at the level of the $\tau$-functions

$$
\tilde{C} t^{\frac{1}{8}\left(\theta_{0}^{2}+\theta_{t}^{2}-\theta_{1}^{2}-\theta_{\infty}^{2}\right)-\frac{1}{2} e_{2}\left[v^{\mathrm{JUE}}\right]-\mu N}(1-t)^{\frac{1}{8}\left(-\theta_{0}^{2}+\theta_{t}^{2}+\theta_{1}^{2}-\theta_{\infty}^{2}\right)-e_{2}^{\prime}\left[v^{\mathrm{JUE}}\right]+\frac{1}{2} e_{2}\left[v^{\mathrm{JUE}}\right]} \tau(t ; \theta) .
$$

Now applying the $t \rightarrow 0$ expansions for $\tau(t ; \theta)$, namely (3.55), with those of $A_{N}(t ;),(2.1)$, we first note that the exponent of the $t$-prefactors must be consistent and this implies

$$
\sigma_{0 t}^{2}=\frac{1}{2}\left(\theta_{0}^{2}+\theta_{t}^{2}+\theta_{1}^{2}+\theta_{\infty}^{2}\right)+2 e_{2}\left[v^{\mathrm{JUE}}\right]=\left(\sum_{i=1}^{4} v_{i}^{\mathrm{JUE}}\right)^{2}=(N-\mu+\bar{\omega})^{2} .
$$

This result applies to all the cases and for definiteness we make the choice of sign outlined in (4.4) (the other choice of sign is essentially equivalent). Turning to the leading analytic term of order $t$ in both expansions we find that its coefficient is given precisely by

$$
\frac{2 \mu(\mu+\omega) N}{N-\mu+\bar{\omega}}
$$

for all three cases upon employing our solution for $\sigma_{0 t}^{2}$. Next we make a comparison of the non-analytic terms in (2.1) and (3.55) and it is here that we have to treat the cases separately. However it is generally true that in the classical situation of the finite rank random matrix ensemble only one of the non-analytic terms is ever present, the other being switched off through the following mechanism. Taking case (A) first our task is to show how the coefficient of the non-analytic term $t^{1+\sigma_{0 t}}$ vanishes and to match the remaining coefficient with that of the application. The identification of $\sigma_{0 t}$ immediately implies $\sigma_{0 t}=\theta_{0}+\theta_{t}$. Therefore

$$
\left[t^{1+\sigma_{0 t}}\right] \propto \frac{1}{\Gamma\left(\frac{\theta_{0}+\theta_{t}-\sigma_{0 t}}{2}\right)}=0 .
$$


From a comparison of the coefficients of the remaining non-analytic term we find precise agreement and this enables us to determine the solution for the monodromy coefficient $s_{0 t}$ in (4.5). For case (B) we note that $\sigma_{0 t}=\theta_{t}-\theta_{0}$ and thus

$$
\left[t^{1+\sigma_{0 t}}\right] \propto \frac{1}{\Gamma\left(\frac{-\theta_{0}+\theta_{t}-\sigma_{0 t}}{2}\right)}=0 .
$$

Similarly we find agreement for the coefficient of the surviving non-analytic term and that enables us to fix the monodromy coefficient as in (4.14). In case (C) we have $\sigma_{0 t}=\theta_{1}-\theta_{\infty}$ and thus

$$
\left[t^{1+\sigma_{0 t}}\right] \propto \frac{1}{\Gamma\left(\frac{-\theta_{\infty}+\theta_{1}-\sigma_{0 t}}{2}\right)}=0 .
$$

Again we find agreement for the coefficient of the $t^{1+\sigma_{0 t}}$ term and conclude that the monodromy coefficient is given by (4.20). For each of the three cases we observe that the Jimbo parameterisation of the monodromy fails (see condition (3.22) however a meaningful result emerges so we conjecture that the Theorem still holds with these relaxations.

Now we make a comparison of the expansions at $t=1$, namely (2.2) and (3.60). Examination of the algebraic prefactor using the relation (4.36) leads us to conclude $\sigma_{t 1}^{2}=\left(2 \mu+2 \omega_{1}\right)^{2}$ in all three cases and we choose the positive sign, as in (4.4). Employing this solution we compute the coefficient of the analytic term is, in all three cases,

$$
\frac{\mu(\bar{\omega}-\omega) N}{2 \mu+2 \omega_{1}},
$$

which is are entirely consistent with that in (2.2). To examine the non-analytic terms we take the three cases separately again. For case (A) we see that the coefficient of the $(1-t)^{1-\sigma_{t 1}}$ vanishes because $\sigma_{t 1}=-\theta_{0}-\theta_{\infty}$ and

$$
\left[(1-t)^{1-\sigma_{t 1}}\right] \propto \frac{1}{\Gamma\left(\frac{\theta_{\infty}+\theta_{0}+\sigma_{t 1}}{2}\right)}=0 .
$$

The coefficients of $(1-t)^{1+\sigma_{t 1}}$ now agree precisely provided we have the solution (4.6) for the monodromy coefficient $s_{t 1}$. For case (B) we have the relation $\sigma_{t 1}=$ $\theta_{1}-\theta_{t}$ and see that

$$
\left[(1-t)^{1-\sigma_{t 1}}\right] \propto \frac{1}{\Gamma\left(\frac{-\theta_{1}+\theta_{t}+\sigma_{t 1}}{2}\right)}=0 .
$$

Again the coefficients of $(1-t)^{1+\sigma_{t 1}}$ agree and the solution (4.15) for $s_{t 1}$ follows. In case $(\mathrm{C})$ we see that $\sigma_{t 1}=\theta_{\infty}-\theta_{0}$ and this ensures

$$
\left[(1-t)^{1-\sigma_{t 1}}\right] \propto \frac{1}{\Gamma\left(\frac{-\theta_{\infty}+\theta_{0}+\sigma_{t 1}}{2}\right)}=0 .
$$

In this case we also find the coefficients of the remaining non-analytic terms are precisely consistent, leading us to deduce the solution (4.21) for $s_{t 1}$. 
It remains to make a comparison of the expansions at $t=\infty$, namely $(2.3)$ and (3.61). Using (4.36) we see the algebraic prefactor implies that $\sigma_{01}^{2}=(N-\mu+\omega)^{2}$, and we choose the positive sign for the exponent. Using this value for $\sigma_{01}$ we compute that the coefficient for the $t^{-1}$ term in all three cases is

$$
\frac{2 \mu(\mu+\bar{\omega}) N}{N-\mu+\omega}
$$

which is consistent with (2.3). To treat the non-analytic terms we take the cases separately. For case (A) we note that $\sigma_{01}=\theta_{t}+\theta_{\infty}$ and this implies

$$
\left[t^{-1-\sigma_{01}}\right] \propto \frac{1}{\Gamma\left(\frac{\theta_{\infty}+\theta_{t}-\sigma_{01}}{2}\right)}=0 .
$$

The coefficient of the $t^{-1+\sigma_{01}}$ term is found to be in agreement with that of (2.3) if we take the solution (4.7) for $s_{01}$. For case (B) the relation is $\sigma_{01}=\theta_{t}-\theta_{\infty}$ and this in turn implies

$$
\left[t^{-1-\sigma_{01}}\right] \propto \frac{1}{\Gamma\left(\frac{-\theta_{\infty}+\theta_{t}-\sigma_{01}}{2}\right)}=0 .
$$

Again exact agreement is found for the other coefficient provided that (4.16) holds. Lastly in case (C) we have the same relation as above and the absence of the $t^{-1-\sigma_{01}}$ term. Examination of the coefficients of $t^{-1+\sigma_{01}}$ then lead us to the solution (4.22).

Now we come to consideration of the connection relation (3.48) for $t=0$ and its two equivalent forms for $t=1, \infty$ with respect to our solutions for the monodromy data. We compute that the three connection relations of either sign decouple into a left-hand side and a right-hand side which vanish separately for all the cases (A), (B) and (C). The left-hand sides for the $t=0$ connection relation vanish because $\theta_{0}+\theta_{t}-\sigma_{0 t}=0$ and $\theta_{\infty}+\theta_{1}+\sigma_{0 t}=2 N$ for case (A), $\theta_{0}-\theta_{t}+\sigma_{0 t}=0$ and $\theta_{0}+\theta_{t}+\sigma_{0 t}=2 N$ for case (B), and $\theta_{\infty}-\theta_{1}+\sigma_{0 t}=0$ and $\theta_{0}+\theta_{t}+\sigma_{0 t}=2 N$ for case $(\mathrm{C})$. Similar reasoning applies to the connection relations at $t=1$ and $t=\infty$. The right-hand sides of the relations vanish identically for both signs with the evaluations of $\sigma_{0 t}, \sigma_{t 1}, \sigma_{01}$ as given in (4.4).

To conclude we compute the monodromy matrices for the three cases and note that case (A), case (B) and case (C) yield the classical monodromy structure of lower triangular matrices, full matrices with one being a signed multiple of the identity, and upper triangular matrices respectively.

Remark 4.1. Our results are consistent with the findings of Mazzocco [20] which state that the classical non-algebraic solutions for $\mathrm{P}_{\mathrm{VI}}$ have either reducible monodromy groups (cases $(\mathrm{A})$ and $(\mathrm{C})$ ) or at least one monodromy matrix is equal to $\pm I$, that is the monodromy group is 1 -smaller (case (B)). Both these cases cover the situation of a one parameter $(N)$ family of classical solutions.

Remark 4.2. We observe that the exponents $\sigma_{0 t}, \sigma_{t 1}, \sigma_{01}$ are not free boundary conditions for classical solutions but are fixed by certain combinations of the formal 
monodromy exponents. Related to this phenomenon is that all the connection relations decouple so that the coefficients of the monodromy coefficients $s_{0 t}, s_{t 1}, s_{01}$ all vanish and thus cannot be determined from these relations. There is a geometrical picture of the classical solutions, which was discussed in relation to Painlevé II by Its and Kapaev [14]. The classical solutions of $\mathrm{P}_{\mathrm{VI}}$ define singular points in the monodromy manifold which are characterised by $\mathfrak{M}=0$ and

$$
\begin{aligned}
& \frac{\partial}{\partial p_{0 t}} \mathfrak{M}=p_{t 1} p_{01}+2 p_{0 t}-p_{0} p_{t}-p_{1} p_{\infty}=0 \\
& \frac{\partial}{\partial p_{t 1}} \mathfrak{M}=p_{0 t} p_{01}+2 p_{t 1}-p_{t} p_{1}-p_{0} p_{\infty}=0 \\
& \frac{\partial}{\partial p_{01}} \mathfrak{M}=p_{0 t} p_{t 1}+2 p_{01}-p_{0} p_{1}-p_{t} p_{\infty}=0 .
\end{aligned}
$$

We verify that these relations are satisfied for the cases (A), (B) and (C).

\section{ACKNOWLEDGEMEnts}

This work was supported by the Australian Research Council.

\section{REFERENCES}

[1] M. Adler and P. van Moerbeke. Hermitian, symmetric and symplectic random ensembles: PDEs for the distribution of the spectrum. Ann. of Math. (2), 153(1):149-189, 2001.

[2] P. Boalch. From Klein to Painlevé via Fourier, Laplace and Jimbo. Proc. London Math. Soc. (3), 90(1):167-208, 2005.

[3] A. Borodin and P. Deift. Fredholm determinants, Jimbo-Miwa-Ueno $\tau$-functions, and representation theory. Comm. Pure Appl. Math., 55(9):1160-1230, 2002.

[4] P. J. Forrester. Log Gases and Random Matrices. http://www.ms .unimelb.edu.au/matpjf/matpjf.html.

[5] P. J. Forrester and N. S. Witte. Application of the $\tau$-function theory of Painlevé equations to random matrices: PIV, PII and the GUE. Comm. Math. Phys., 219(2):357-398, 2001.

[6] P. J. Forrester and N. S. Witte. Application of the $\tau$-function theory of Painlevé equations to random matrices: $\mathrm{P}_{\mathrm{V}}, \mathrm{P}_{\mathrm{III}}$, the LUE, JUE, and CUE. Comm. Pure Appl. Math., 55(6):679$727,2002$.

[7] P. J. Forrester and N. S. Witte. Application of the $\tau$-function theory of Painlevé equations to random matrices: $\mathrm{P}_{\mathrm{VI}}$, the JUE, CyUE, cJUE and scaled limits. Nagoya Math. J., 174:29$114,2004$.

[8] P. J. Forrester and N. S. Witte. Discrete Painlevé equations for a class of $P_{\mathrm{VI}} \tau$-functions given as $\mathrm{U}(N)$ averages. Nonlinearity, 18(5):2061-2088, 2005.

[9] P. J. Forrester and N. S. Witte. Bi-orthogonal Polynomials on the Unit Circle, regular semiclassical Weights and Integrable Systems. Construct. Approx., 24(2):201-237, 2006.

[10] P.J. Forrester and N.S. Witte. Boundary conditions associated with the Painlevé III' and V evaluations of some random matrix averages. J. Phys. A: Math. Gen., 39(28):8983-8995, 2006.

[11] R. Fuchs. Über lineare homogene Differentialgleichungen zweiter Ordnung mit drei im Endlichen gelegenen wesentlich singulären Stellen. Comptes Rendus de l'Académie des Sciences de Paris, 141:555-558, 1905. 
[12] D. Guzzetti. The elliptic representation of the general Painlevé VI equation. Comm. Pure Appl. Math., 55(10):1280-1363, 2002.

[13] L. Haine and J.-P. Semengue. The Jacobi polynomial ensemble and the Painlevé VI equation. J. Math. Phys., 40:2117-2134, 1999.

[14] A. R. Its and A. A. Kapaev. The irreducibility of the second Painlevé equation and the isomonodromy method. In Toward the exact WKB analysis of differential equations, linear or non-linear (Kyoto, 1998), pages 203, 209-222. Kyoto Univ. Press, Kyoto, 2000.

[15] M. Jimbo. Monodromy problem and the boundary condition for some Painlevé equations. Publ. Res. Inst. Math. Sci., 18(3):1137-1161, 1982.

[16] H. Kimura and K. Okamoto. On the polynomial Hamiltonian structure of the Garnier systems. J. Math. Pures Appl. (9), 63(1):129-146, 1984.

[17] A. V. Kitaev. An Isomonodromy Cluster of Two Regular Singularities. Sfb 288 Preprint No. 149, 1994.

[18] A. P. Magnus. Painlevé-type differential equations for the recurrence coefficients of semiclassical orthogonal polynomials. In Proceedings of the Fourth International Symposium on Orthogonal Polynomials and their Applications (Evian-Les-Bains, 1992), volume 57, pages 215-237, 1995.

[19] J. Malmquist. Sur les équations différentielles du second ordre dont l'intégrale générale a ses points critiques fixes. Arkiv Mat., Astron. Fys., 18(8):1-89, 1922.

[20] M. Mazzocco. The geometry of the classical solutions of the Garnier systems. Int. Math. Res. Not., (12):613-646, 2002.

[21] J.-M. Normand. Calculation of some determinants using the $s$-shifted factorial. J. Phys. A, 37(22):5737-5762, 2004.

[22] K. Okamoto. Isomonodromic deformation and Painlevé equations, and the Garnier system. J. Fac. Sci. Univ. Tokyo Sect. IA Math., 33(3):575-618, 1986.

[23] K. Okamoto. Studies on the Painlevé equations. I. Sixth Painlevé equation $P_{\mathrm{VI}}$. Ann. Mat. Pura Appl. (4), 146:337-381, 1987.

[24] C. A. Tracy and H. Widom. Fredholm determinants, differential equations and matrix models. Comm. Math. Phys., 163(1):33-72, 1994.

[25] N. S. Witte and P. J. Forrester. Gap probabilities in the finite and scaled Cauchy random matrix ensembles. Nonlinearity, 13(6):1965-1986, 2000.

Department of Mathematics and Statistics, University of Melbourne, Victoria 3010, Australia

E-mail address: p.forrester@ms.unimelb.edu.au; n.witte@ms.unimelb.edu.au 